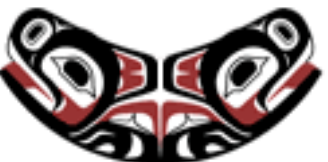

UW Biostatistics Working Paper Series

4-8-2004

\title{
Evaluating Markers for Selecting a Patient's Treatment
}

\author{
Xiao Song \\ University of Washington, songx@u.washington.edu \\ Margaret S. Pepe \\ University of Washington, mspepe@u.washington.edu
}

\section{Suggested Citation}

Song, Xiao and Pepe, Margaret S., "Evaluating Markers for Selecting a Patient's Treatment" (April 2004). UW Biostatistics Working Paper Series. Working Paper 206.

http://biostats.bepress.com/uwbiostat/paper206

This working paper is hosted by The Berkeley Electronic Press (bepress) and may not be commercially reproduced without the permission of the copyright holder.

Copyright $(\odot) 2011$ by the authors 


\section{Introduction}

For many medical conditions there are several treatment options available to the patient. For example, carpal tunnel syndrome (CTS) can be treated surgically or with conservative therapy that includes physical therapy and anti-inflammatory medications. Conservative therapy is less invasive and less expensive than surgery and is preferable from those points of view. However, it is probably not as effective as surgery, at least in some patients. A simple randomized trial to compare surgery and conservative therapy is currently underway at the University of Washington. All patients in the trial receive magnetic resonance neurographic imaging (MRNI) at baseline. Although the MRNI does not influence treatment within the clinical trial, it is thought that the MRNI score may be an indicator of which patients are likely to benefit more from surgery and could be used in the future to select patients for surgery versus conservative therapy.

This paper addresses statistical techniques to evaluate the capacity of a measure, denoted by $Y$, to assist in treatment selection. The issue is important not only for our study but more generally and particularly in this era of rapidly expanding biotechnology. One of the promises of new biotechnologies, including gene expression arrays and imaging modalities, is to provide information for the purpose of selecting optimal treatment of disease (ElmerDewitt et al., 2001). It is clearly important that appropriate statistical techniques are in place to critically evaluate the technologies before they are adopted for widespread use.

In Section 2 we present a graphical display, the selection impact (SI) curve that directly describes the performance of the measure $Y$ for treatment selection. We contrast this with existing approaches. Next we propose methods for estimating the SI curve with data from a randomized trial. A nonparametric estimator described in Section 3 and a parametric estimator described in Section 4 are contrasted in the following two sections, using asymptotic theory and small sample simulation studies. Data, simulated to reflect anticipated results 
from the CTS randomized trial, are analyzed in Section 7. We close in Section 8 with suggestions for future research.

\section{The Selection Impact Curve}

1. Definition and Purpose

First we define some notation. Let $T$ be a binary variable that denotes treatment. For simplicity we suppose that there are two treatment options available in the population, $A$ and $B$. Let $T=1$ for $A$ and $T=0$ for $B$. Using the notion of potential responses (Rubin, 1974, 1978; Holland, 1986), for $t=0,1$, we define $D^{t}$ as the response if a subject receives treatment $T=t$. It is impossible to observe $D^{0}$ and $D^{1}$ on the same subject; instead, $D=T D^{1}+(1-T) D^{0}$ is observed. In this paper, we consider the case that $D^{t}$ is dichotomous, $D^{t}=1$ for success and $D^{t}=0$ for failure. Extensions to nondichotomous outcomes are discussed in Section 8. We assume that an individual's response to treatment does not depend on the assignment mechanism or the assignments or responses of other individuals to treatments, i.e., the SUTVA assumption of Rubin (1986) holds. The measure $Y$, potentially used in the future for selecting patient treatment, is measured on a continuous scale and larger values of $Y$ are potentially associated with better performance of treatment $A$ versus $B$. ( $Y$ can be recoded if necessary to achieve this).

Consider the following treatment policy based on a patient's marker measure $Y$ exceeding a threshold to determine which treatment the patient receives:

$$
\begin{aligned}
& Y>c: \quad \text { select treatment } A, \\
& Y \leq c: \quad \text { select treatment } B .
\end{aligned}
$$

We focus on simple thresholding criteria because, at least in our experience, criteria of this 
form are most often implemented in clinical protocols. (Consider some common examples such as serum creatine $>1.3 \mathrm{mg} / \mathrm{dL}$, cholesterol $>200 \mathrm{mg} / \mathrm{dL}$, fasting blood glucose $>126$ $\mathrm{mg} / \mathrm{dL}$ and serum PSA $>4.0 \mathrm{ng} / \mathrm{mL}$ ). The population response rate corresponding to this policy is

$$
\begin{aligned}
\theta & =\mathrm{P}[D=1 \mid(Y>c \text { and } T=1) \text { or }(Y<c \text { and } T=0)] \\
& =\mathrm{P}\left[D^{1}=1 \mid Y>c\right] \mathrm{P}[Y>c]+\mathrm{P}\left[D^{0}=1 \mid Y<c\right] \mathrm{P}[Y<c]
\end{aligned}
$$

That is, $\theta$ is the proportion of subjects in the population who respond if the treatment policy in effect is to assign a subject to treatment $A$ if his marker value exceeds $c$ but to assign him to treatment $B$ otherwise. Observe that when $c=-\infty$, the policy is that all patients receive treatment $A$ and none receive $B$, while for $c=\infty$ all patients receive treatment $B$. As $c$ increases from $-\infty$ to $\infty$, the proportion of subjects assigned to treatment $B$ increases from 0 to 1 . Figure 1 displays a schematic illustration of the population response rate as $c$ varies from $-\infty$ to $+\infty$. In the illustration, the overall response rate is higher when all patients are on treatment $A$ than when all are on treatment $B$. The curve indicates however that a policy that assigns $40 \%$ of patients to treatment $B$ and $60 \%$ to treatment $A$ on the basis of $Y$, can perform almost as well as one that assigns $100 \%$ of patients to treatment $A$. If treatment $B$ is substantially less expensive or invasive than $A$, as is the case in the CTS study, then this represents a better treatment policy.

The SI curve shows the impact on population response rates of treatment selection criteria based on $Y$ exceeding a threshold. We show the curve, $\theta(v)$, as a function of $v=\mathrm{P}[Y<c]$ rather than as a function of $c$ itself. There are two reasons for this. First, in evaluating a treatment policy of this sort, it will be important to know the fractions of subjects potentially assigned to treatment $A$ versus $B$ by the policy, $1-v$ and $v$, respectively. Indeed it is the 
trade-off between the overall population response rate achieved and $v$ that is of key interest. If satisfactory operating points are found, $(v, \theta(v))$, then one can ascertain the corresponding thresholds to implement the policy. Second, the display on this scale allows one to compare policies based on different markers. SI curves for two hypothetical markers are shown in the right panel of Figure 1. The measurement units of the two markers, $Y_{1}$ and $Y_{2}$, say, are irrelevant for the purposes of comparing selection policies based on them. Rather, policies that assign equal fractions of the patient populations to treatment $B$ more naturally compare the markers. We see in Figure 1 that when the thresholds for the two markers, $c_{1}$ and $c_{2}$, are chosen so that $\mathrm{P}\left[Y_{1}<c_{1}\right]=\mathrm{P}\left[Y_{2}<c_{2}\right]=v$, the marker $Y_{1}$ yields better performance. In summary, the SI curve provides a natural common scale for comparing response rates achieved with treatment selection policies based on different markers.

Receiver operating characteristic curves that are used to evaluate and compare operating characteristic of diagnostic tests are motivated by similar notions (Pepe 2003). Test positive criteria are defined by thresholding the test result and consequent sensitivity and specificity values are plotted. This provides a practically relevant scale for evaluating tests and an appropriate common scale for comparing tests.

In summary, we propose the SI curve as a simple graphical device that shows the population response rates potentially achieved with treatment assignment policies based on the marker exceeding a threshold or not. It can be used to determine if, by using the marker, there is potential to treat fewer patients with an invasive expensive treatment while maintaining the overall proportion of subjects responding at or above that achieved by sending all patients to that treatment. It can suggest an optimal threshold value. Finally, it provides a natural common scale on which to compare different treatment selection markers. 
A traditional statistical approach to evaluating differential treatment benefit is to use binary regression models for the response variable $D$ with treatment, $T$, and the measure, $Y$, as covariates. See Byar (1985) and references therein. In this traditional framework an interaction between $Y$ and $T$ is interpreted to mean that $Y$ informs about the relative performance of the treatments. Suppose for example we fit the model

$$
\operatorname{logitP}[D=1 \mid Y, T]=\alpha_{1}+\alpha_{2} Y+\alpha_{3} T+\alpha_{4} Y T
$$

and consider the coefficient $\alpha_{4}$ for interaction. The quantity $\exp \left(\alpha_{4}\right)$ is the increase in the odds ratio associated with treatment $A$ versus $B$ per unit increase in $Y$. This seems a few steps removed from quantifying the potential impact on the population of using $Y$ to select treatment. The SI curve does this more directly. Moreover, one can find settings where there is no statistical interaction between $Y$ and $T$ in the regression model, but the marker is informative (see setting 7 of Figure 2). This is because the definition of interaction in a regression model depends on the metric on which the linear predictor is defined. Thus data that yield an interaction when a logistic link function is used may yield no interaction when another link function is used. The SI curve does not depend on the somewhat arbitrary definition of interaction that the regression framework does. It simply shows the population response rates according to treatment selection criteria based on $Y$ exceeding a threshold.

\section{Other Features of the SI Curve}

We write $y_{v}$ for the $v^{t h}$ quantile of $Y$ in the population so that $v=\mathrm{P}\left[Y<y_{v}\right]$. The SI curve also shows the following entities that are important measures of the value of the 
treatment policy that uses the threshold $y_{v}$ for deciding on patient treatment:

$$
\begin{aligned}
d^{+}(v) & =\mathrm{P}\left[D=1 \mid Y>y_{v}, T=1\right]-\mathrm{P}\left[D=1 \mid Y>y_{v}, T=0\right] \\
& =\mathrm{P}\left[D^{1}=1 \mid Y>y_{v}\right]-\mathrm{P}\left[D^{0}=1 \mid Y>y_{v}\right], \\
d^{-}(v) & =\mathrm{P}\left[D=1 \mid Y<y_{v}, T=0\right]-\mathrm{P}\left[D=1 \mid Y<y_{v}, T=1\right] \\
& =\mathrm{P}\left[D^{0}=1 \mid Y<y_{v}\right]-\mathrm{P}\left[D^{1}=1 \mid Y<y_{v}\right] .
\end{aligned}
$$

The first, $d^{+}(v)$, relates to the advantage of assigning treatment $A$ versus $B$ to patients who score above the $v^{\text {th }}$ quantile of $Y$. Correspondingly $d^{-}(v)$ is the difference in response rates for patients below the $v^{t h}$ quantile when they are given treatment $B$ versus $A$. Ideally, the threshold $y_{v}$ is chosen so that both groups of patients benefit by the policy, i.e., $d^{+}(v)>0$ and $d^{-}(v)>0$. To see $d^{+}(v)$ from the SI curve, observe from (1) that

$$
\theta(v)=(1-v) \mathrm{P}\left[D^{1}=1 \mid Y>y_{v}\right]+v P\left[D^{0}=1 \mid Y<y_{v}\right]
$$

Moreover, we can write

$$
\begin{aligned}
\theta(0) & =\mathrm{P}\left[D^{1}=1\right]=\mathrm{P}\left[D^{1}=1, Y>y_{v}\right]+\mathrm{P}\left[D^{1}=1, Y<y_{v}\right] \\
& =(1-v) \mathrm{P}\left[D^{1}=1 \mid Y>y_{v}\right]+v P\left[D^{1}=1 \mid Y<y_{v}\right]
\end{aligned}
$$

It follows that

$$
\theta(v)-\theta(0)=v d^{-}(v)
$$

Similar arguments show that

$$
\theta(v)-\theta(1)=(1-v) d^{+}(v)
$$

Thus the distances between the SI curve and the upper and lower horizontal lines in Figure 1, show separately the potential consequences of the treatment policy to the 2 groups of patients 
with marker measurements $Y$ that are above and below the threshold $y_{v}$. In the left panel of Figure 1, using the threshold $y_{0.4}$ to decide on treatment, we see that the response rate of subjects with $Y>y_{0.4}$ is greatly improved with treatment $A$ versus treatment $B, d^{+}(0.4)=$ $\frac{\theta(0.4)-\theta(1)}{1-0.4}=0.69$. On the other hand, for the group with $Y<y_{0.4}$ the response rate with treatment $B$ is reduced but not by very much relative to treatment $A, d^{-}(0.4)=\frac{\theta(0.4)-\theta(0)}{0.4}=$ -0.09 .

An uninformative marker is one that does not identify subsets of patients that benefit more than others do by their assigned treatment. That is, an uninformative marker is defined as one for which

$$
\mathrm{P}\left[D^{1}=1 \mid Y>y_{v}\right]-\mathrm{P}\left[D^{0}=1 \mid Y>y_{v}\right]=\mathrm{P}\left[D^{1}=1\right]-\mathrm{P}\left[D^{0}=1\right]
$$

for all $v$. Thus, for the uninformative marker $d^{+}(v)=\theta(0)-\theta(1)$. Similarly, for the uninformative marker, $d^{-}(v)=\theta(1)-\theta(0)$. Since in general $\theta(v)=\theta(1)+(1-v) d^{+}(v)$ it follows that $\theta(v)$ is a straight line connecting $\theta(1)$ to $\theta(0)$ for the uninformative marker. This serves as a baseline SI curve against which others can be compared. Observe that the uninformative marker may be associated with treatment response. It simply does not inform about which patients are likely to benefit more than the average from treatment $A$ versus $B$ or vice versa.

Our SI curve displays $d^{+}(v)$ and $d^{-}(v)$, the differences in response rates with treatments $A$ and $B$ for the population that meets the criterion $Y>y_{v}$ and that which does not. In setting forth a treatment policy for a population, consideration of the population as a whole that meets the criterion (or not) is most relevant. Nevertheless, an individual patient with marker value $Y=y$, will be more interested in his/her own probabilities of response with 
the two treatments. We write

$$
d(v)=\mathrm{P}\left[D=1 \mid Y=y_{v}, T=1\right]-\mathrm{P}\left[D=1 \mid Y=y_{v}, T=0\right] .
$$

With knowledge of the risks and costs associated with treatments $A$ versus $B$ and the difference in response probabilities $d(v)$, an individual patient and caregiver may decide on which treatment to select. However, in our experience such individual decision making is a luxury not always afforded by the healthcare system and, as mentioned earlier, simple dichotomous criteria such as $Y$ exceeding a percentile threshold are often used to make medical decisions. Policy makers will be interested in the impact of such criteria on the overall response probabilities, i.e., the quantities shown in the SI curve, $\theta(v), d^{+}(v)$ and $d^{-}(v)$.

In addition, we will see that $d(v)$ is much more difficult to estimate from data than are the cumulative versions

$$
d^{+}(v)=(1-v)^{-1} \int_{v}^{1} d(v) d v \quad \text { and } \quad d^{-}(v)=-v^{-1} \int_{0}^{v} d(v) d v
$$

Estimating $d(v)$ is akin to estimating a density while estimating $d^{+}(v), d^{-}(v)$ and $\theta(v)$, is akin to the much simpler task of estimating a cumulative distribution function. For example, a completely nonparametric estimator of $\theta(v)$ is proposed in Section 3 while the nonparametric estimation of $d(v)$ requires smoothing techniques as shown in the data analysis of Section 7 . We also discuss parametric estimation of $\theta(v)$ in Section 4 using parametric modeling of $\mathrm{P}[D=1 \mid T, Y]$ as the key stepping stone. In essence, we estimate the components of $d(v)$, $\mathrm{P}[D=1 \mid T=1, Y]$ and $\mathrm{P}[D=1 \mid T=0, Y]$, parametrically in this approach. Although somewhat more efficient than the nonparametric method (Table 1) and yielding $d(v)$ as a byproduct, we do not ultimately advocate the parametric approach because it can give misleading results under a misspecified model. 


\section{Nonparametric Estimation}

We now turn to estimation of $\theta(v)$. Data from a randomized trial conducted in the population can be used for estimation if some intuitively reasonable conditions are met. These include: (i) subjects enrolled in the trial are a simple random sample from the population of interest; (ii) response to treatment observed for an individual in the trial, $D_{i}$, reflects the potential response of an individual in the population assigned to that treatment, as discussed in Section 2. That is, $D_{i}=T D_{i}^{1}+(1-T) D_{i}^{0}$; (iii) treatment assignment or care of the patient in the trial does not depend on his marker value $Y$ measured at enrollment. We discuss these conditions later in Section 8 but for now proceed with estimation methods assuming that they hold.

Suppose that we have independent observations $\left(D_{i}, Y_{i}, T_{i}\right)$ for $i=1, \ldots, n$ subjects. Observe that

$$
\theta(v)=(1-v) \frac{G_{1}^{D}\left(y_{v}\right)}{G_{1}\left(y_{v}\right)}+v \frac{G_{2}^{D}\left(y_{v}\right)}{G_{2}\left(y_{v}\right)},
$$

where $G_{1}^{D}(y)=\operatorname{Pr}(D=1, Y>y, T=1), G_{1}(y)=\operatorname{Pr}(Y>y, T=1), G_{2}^{D}(y)=\operatorname{Pr}(D=$ $1, Y \leq y, T=0)$, and $G_{2}(y)=\operatorname{Pr}(Y \leq y, T=0)$. Thus, substituting the empirical estimators for the probabilities, a natural nonparametric estimator for $\theta(v)$ is

$$
\widehat{\theta}_{n p}(v)=(1-v) \frac{G_{1 n}^{D}\left(\hat{y}_{v}\right)}{G_{1 n}\left(\hat{y}_{v}\right)}+v \frac{G_{2 n}^{D}\left(\hat{y}_{v}\right)}{G_{2 n}\left(\hat{y}_{v}\right)},
$$

where $\hat{y}_{v}=F_{n}^{-1}(v), F_{n}(y)=n^{-1} \sum_{i=1}^{n} I\left(Y_{i} \leq y\right)$ is the empirical distribution function for $F(y)=\operatorname{Pr}(Y \leq y)$, and $G_{1 n}^{D}(y)=n^{-1} \sum_{i=1}^{n} I\left\{D_{i}=1, Y_{i}>\hat{y}_{v}, T_{i}=1\right\}, G_{1 n}(y)=$ $n^{-1} \sum_{i=1}^{n} I\left\{Y_{i}>\hat{y}_{v}, T_{i}=1\right\}, G_{2 n}^{D}(y)=n^{-1} \sum_{i=1}^{n} I\left\{D_{i}=1, Y_{i} \leq \hat{y}_{v}, T_{i}=0\right\}, G_{2 n}(y)=$ $n^{-1} \sum_{i=1}^{n} I\left\{Y_{i} \leq \hat{y}_{v}, T_{i}=0\right\}$ are the empirical estimators for $G_{1}^{D}(y), G_{1}(y), G_{2}^{D}(y)$ and $G_{2}(y)$, respectively. Basically, we determine the empirical quantile $\hat{y}_{v}$ and calculate the proportion 
of subjects on treatment $A$ with $Y>\hat{y}_{v}$ who respond and the proportion on treatment $B$ with $Y \leq \hat{y}_{v}$ who respond. The weighted average is the empirical nonparametric estimator of $\theta(v)$.

Using the empirical process theory, we can show that $\sqrt{n}\left\{\widehat{\theta}_{n p}(v)-\theta(v)\right\}$ converges to a Gaussian process $\mathbb{Z}(v)$ with $\operatorname{cov}\left\{\mathbb{Z}\left(v_{1}\right), \mathbb{Z}\left(v_{2}\right)\right\}=A^{T}\left(v_{1}\right) \Sigma\left(y_{v_{1}}, y_{v_{2}}\right) A\left(v_{2}\right)$, where $A(\cdot)$ and $\Sigma(\cdot)$ are given in Appendix A. The asymptotic variance for $\widehat{\theta}_{n p}(v)$ therefore is $V_{n p}(v)=$ $n^{-1} A^{T}(v) \Sigma\left(y_{v}, y_{v}\right) A(v)$. In Section 5 we calculate the large sample variance of $\widehat{\theta}_{n p}(v)$ in various settings using this expression. In applications, we can use the bootstrap method to calculate the standard error and confidence band for $\theta(v)$ for $v \in[a, b](0<a<b<1)$. A detailed proof and arguments to justify bootstrapping are given in Appendix A.

\section{Parametric Estimation}

An alternative approach to estimating $\theta(v)$ is based on regression modeling. Suppose we use a model of the form

$$
\operatorname{logit} \mathrm{P}\left[D=1 \mid Y=y_{v}, T\right]=\operatorname{logit} \mathrm{P}[D=1 \mid F(Y)=v, T]=\alpha^{T} R(v, T)
$$

where $R(v, T)$ is a $q$-dimensional function of $v$ and $T$. It is useful to write the model in terms of $v$, since $\theta(v)$ is considered a function of $v$ for the reasons mentioned earlier. For example we might use the model

$$
\operatorname{logit} \mathrm{P}[D=1 \mid F(Y)=v, T]=\alpha_{1}+\alpha_{2} R(v)+\alpha_{3} T+\alpha_{4} R(v) T
$$

where $R(v)$ is some function of $v$. We can write

$$
\mathrm{P}\left[D=1 \mid Y>y_{v}, T=1\right]=\mathrm{P}[D=1 \mid F(Y)>v, T=1]=\frac{\int_{v}^{1} \mathrm{P}[D=1 \mid F(Y)=w, T=1] d w}{(1-v)}
$$

and

$$
\mathrm{P}\left[D=1 \mid Y<y_{v}, T=0\right]=\frac{\int_{0}^{v} \mathrm{P}[D=1 \mid F(Y)=w, T=0] d w}{v} .
$$


Therefore if estimates of $\alpha$ are available we can substitute them into the expression for $\theta(v)$

$$
\theta(v)=\int_{v}^{1}\left(1+\exp \left\{-\alpha^{T} R(w, 1)\right\}\right)^{-1} d w+\int_{0}^{v}\left(1+\exp \left\{-\alpha^{T} R(w, 0)\right\}\right)^{-1} d w .
$$

One possibility is to use the maximum likelihood estimates of $\alpha$ based on the model (2), denoted by $\widehat{\alpha}$. The corresponding estimator for $\theta(v)$ is denoted $\widehat{\theta}_{p}(v)$. Note that this assumes that $F$ is known in advance and can be used to calculate $v_{i}=F\left(Y_{i}\right)$ in fitting the model (2). More often the distribution function $F$ will be estimated empirically from the $n$ observations available. We write $\widetilde{\alpha}$ for the estimator that solves the likelihood score equations but with $\widehat{v}_{i}=F_{n}^{*}\left(Y_{i}\right)=\frac{n}{n+1} F_{n}\left(Y_{i}\right)$ substituted for $v_{i}=F\left(Y_{i}\right)$. The resulting estimator of $\theta(v)$ is denoted by $\widetilde{\theta}_{p}(v)$.

Now we consider the asymptotic properties of the estimators. We show in Appendix B that $\sqrt{n}\left\{\widehat{\theta}_{p}(v)-\theta(v)\right\}$ and $\sqrt{n}\left\{\widetilde{\theta}_{p}(v)-\theta(v)\right\}$ converge to Gaussian processes with mean 0 and covariance structures $\Lambda\left(v_{1}, v_{2} ; V_{\alpha}\right)$ and $\Lambda\left(v_{1}, v_{2} ; \widetilde{V}_{\alpha}\right)$, respectively, where $\Lambda(\cdot), V_{\alpha}$ and $\widetilde{V}_{\alpha}$ are given in Appendix B. Moreover, and not surprisingly, $\Lambda\left(v, v ; \widetilde{V}_{\alpha}\right)>_{p d} \Lambda\left(v, v ; V_{\alpha}\right)$; that is $\Lambda\left(v, v ; \widetilde{V}_{\alpha}\right)-\Lambda\left(v, v ; V_{\alpha}\right)$ is positive definite. By analogy to the nonparametric case, in practice, we can use the bootstrap method to compute estimates of the standard errors and confidence bands for $\theta(v)$.

\section{Asymptotic Relative Efficiencies}

The three estimators $\widehat{\theta}_{n p}, \widetilde{\theta}_{p}$, and $\widehat{\theta}_{p}$ require increasingly stronger assumptions to hold. $\widehat{\theta}_{p}$ assumes that the regression model (2) holds and that $F$ is known. Although $\widetilde{\theta}_{p}$ requires $(2)$, it does not need $F$ to be known since it uses the data to estimate $F$. Finally, $\widehat{\theta}_{n p}$, the empirical estimator, is completely nonparametric. Using the asymptotic variance expressions we calculated the relative efficiencies of the estimators in settings where all three are consistent, i.e., with (2) holding and $F$ correctly specified for $\widehat{\theta}_{p}$. As expected, the estimators that 
assume more are more efficient asymptotically (Table 1).

Figure 2 displays the SI curves for the various settings considered. The settings differ by varying the coefficients $\left(\alpha_{1}, \alpha_{2}, \alpha_{3}, \alpha_{4}\right)$ and the function $R(v)$ in the model (3) for the response probability $\mathrm{P}[D=1 \mid F(Y)=v, T]$, where $v$ is the percentile value of $Y$. It appears that although treatment $A$ is better overall, we do not need to treat all patients with treatment $A$ in some of the settings. For example, in setting 2 , with $R(v)=\Phi^{-1}(v)$, and $\alpha=(0,1,1,1)$, about $30 \%$ can be treated with treatment $B$. In setting 3 , with $R(v)=10 \Phi^{-1}(v)$, treating subjects who score at or below the $40^{\text {th }}$ percentile of $Y$ with treatment $B$ yields an overall response rate that slightly exceeds that when all patients are given treatment $A$.

The most important comparison between the estimators is between $\widehat{\theta}_{n p}$ and $\widetilde{\theta}_{p}$ since $F$ will almost never be known in practice. Efficiency gains of $20-30 \%$ are achieved routinely with $\widetilde{\theta}_{p}$ relative to $\widehat{\theta}_{n p}$ in the scenarios we studied, although greater and lesser gains were also seen. Interestingly for the parametric estimator, knowledge of $F$ further increases its efficiency, sometimes substantially. When $R(v)=10 \Phi^{-1}(v)$ for example, $\widehat{\theta}_{p}$ is generally more than two times as efficient as $\widetilde{\theta}_{p}$. This suggests that in practice parametric estimators of $F$ may yield more efficient estimators of $\theta_{p}(v)$ than that we employed, which are based on the empirical estimator of $F$.

\section{Simulations}

To assess the performance of the estimators in moderate sample sizes we conducted extensive simulation studies. We generated data for $n=200$ subjects in a randomized trial with $\mathrm{P}[T=1]=0.5$ and response variable $D$ from the logistic model $(3)$ with $R(v)=v$ and $R(v)=\Phi^{-1}(v)$, and $\alpha=(0,1,1,1)$. Again we refer to Figure 2 for the corresponding SI functions. The estimated standard errors were computed by the bootstrap method using 
100 resampled data sets. For each estimator, 95\% Wald confidence intervals were computed based on the logit transformation. The results are shown in Table 2. All the estimators exhibit negligible bias. In addition the estimated standard errors track the true standard deviations of the estimators well. Therefore the coverage probabilities are close to their nominal levels. We are comfortable recommending that normal theory inference can be used with any of the estimators. Interestingly, although we used bootstrap estimates of standard errors we note that they are close to the asymptotic theory based values. Thus conclusions about the relative efficiencies of the estimators applied to moderate sized datasets are the same as conclusions stated earlier in Section 5 based on asymptotic theory.

\section{Application to CTS Data}

The CTS study seeks to enroll 200 patients randomized to either median nerve decompressive surgery or conservative therapy with splinting, physical therapy and nonsteroid anti-inflammatory drugs (NSAIDS). Subjects for the study are nonpregnant, 18 years of age or older with no more than 4 months of wrist pain characteristic of CTS. To be eligible for randomization they must have failed to respond adequately to a 2 -week course of minimal conservative therapy with splinting and NSAIDS. Three clinical sites in Seattle are currently enrolling subjects. The primary outcome measure is the functional status index derived from the Carpal Tunnel Syndrome Assessment Questionnaire (CTSAQ) at 12 months after randomization compared with baseline. The change in functional status will be dichotomized as indicating a clinically meaningful improvement or not.

At enrollment individuals undergo MRNI of the median nerve, the results of which are blinded to the patient and personnel involved with his medical care during the trial. The early stages of CTS are thought to be caused by compression of the median nerve by fluid 
accumulating in the narrow space around it. Flatness of the nerve observed on MRNI is thought to be a good marker for patients likely to benefit from surgery that cuts ligaments around the nerve allowing space for it to decompress. This MRNI "flatness" score is measured on a continuous scale and constitutes the proposed treatment selection marker $Y$ to be studied after the trial is completed.

Since the CTS trial is not yet completed, we performed a simulation to reflect the sort of dataset that might occur. Responses were generated from the following model: $\operatorname{logitP}[D=$ $\left.1 \mid T, Y=y_{v}\right]=\alpha_{1}+\alpha_{2} v+\alpha_{3} T+\alpha_{4} T v I(v>0.5)$ with $\alpha_{1}=\operatorname{logit}(0.3), \alpha_{2}=\operatorname{logit}(0.1)-$ $\operatorname{logit}(0.3), \alpha_{3}=0$ and $\alpha_{4}=\operatorname{logit}(0.8)-\left(\alpha_{1}+\alpha_{2}\right)$. This model stipulates that subjects have poorer response rates with conservative therapy if their MRNI scores are high. On conservative therapy a subject with the lowest possible MRNI score $(v=0)$ has a $30 \%$ chance of response while a subject with the highest $(v=1)$ has a $10 \%$ chance of response. On the other hand, surgery works very well for subjects with high MRNI scores. A subject with the highest possible score $(v=1)$ has a response rate of $80 \%$. Subjects with MRNI scores above the median benefit from surgery, with the benefit being an increasing function of $Y$.

The true SI function derived from this model is shown in Figure 3. Estimates of it calculated from the simulated data are also shown. The nonparametric estimate conveys the message that one can assign about $50 \%$ with the lowest values of the MRNI to conventional therapy, and retain the response rate in the population at about that achieved by sending all subjects to surgery. The parametric estimator $\tilde{\theta}_{p}$ that correctly specified the model for $\mathrm{P}\left[D=1 \mid T, Y=y_{v}\right]$ conveys the same result. It is essentially a smoothed version of $\widehat{\theta}_{n p}$. Interestingly the pointwise confidence intervals and confidence bands obtained with 
$\widetilde{\theta}_{p}$ are not substantially more narrow than those of the nonparametric estimator. That is, there is not much to be gained in this example by using the parametric approach except an esthetically more pleasing smooth curve. We fit a second parametric estimator $\widetilde{\theta}_{p}^{*}$ to the data where the model for $\mathrm{P}\left[D=1 \mid T, Y=y_{v}\right]$ was misspecified as $\operatorname{logit} \mathrm{P}\left[D=1 \mid T, Y=y_{v}\right]=$ $\alpha_{1}+\alpha_{2} v+\alpha_{3} T+\alpha_{4} v T$. This estimator is clearly biased and demonstrates the reliance of the parametric method on correct model specification. In this dataset the nonparametric approach is probably best.

Figure 4 illustrates the difference measures $d^{+}(v)$ and $d^{-}(v)$ defined in Section 2. The measures can also be inferred from Figure 3. Estimators are calculated using the relationships $d^{+}(v)=\{\theta(v)-\theta(1)\} /(1-v)$ and $d^{-}(v)=\{\theta(v)-\theta(0)\} / v$ and substituting estimators of $\theta(v), \theta(1)$ and $\theta(0)$. It is not surprising that the nonparametric estimator of $d^{+}(v)$ is unstable for $v$ close to 1 , since there are very few observations for $Y>y_{v}$. A similar phenomenon exists for the nonparametric estimator of $d^{-}(v)$ for $v$ close to 0 . Since $d^{+}(v)$ is positive and $d^{-}(v)$ is negative for $v>0.5$, it is clearly advantageous to treat subjects with MRNI values above the median with surgery rather than with conventional therapy. We see that the gain is more for subjects with the highest MRNI values, i.e. for large $v$. On the other hand $d^{-}(v)$ is approximately 0 for $v<0.5$, so treating subjects with MRI values below the median with conventional therapy is as good as treating them with surgery.

In Figure 5 we display estimators of $d(v)=\mathrm{P}\left[D=1 \mid Y=y_{v}, T=1\right]-\mathrm{P}[D=1 \mid Y=$ $\left.y_{v}, T=0\right]$ that conditions on an individual's marker value rather than on their meeting the dichotomous treatment assignment criterion $Y>y_{v}$ or $Y<y_{v}$. Noting that $d(v)=\frac{d \eta(v)}{d v}$, where $\eta(v)=-v d^{-}(v)=v\left\{\frac{1-G_{1}^{D}\left(y_{v}\right)}{1-G_{1}\left(y_{v}\right)}-\frac{G_{2}^{D}\left(y_{v}\right)}{G_{2}\left(y_{v}\right)}\right\}$, a nonparametric estimator of $d(v)$ is $(2 \tau)^{-1}\{\hat{\eta}(v+\tau)-\hat{\eta}(v-\tau)\}$ with $\hat{\eta}(v)=v\left\{\frac{1-G_{1 n}^{D}\left(\hat{y}_{v}\right)}{1-G_{1 n}\left(\hat{y}_{v}\right)}-\frac{G_{2 n}^{D}\left(\hat{y}_{v}\right)}{G_{2 n}\left(\hat{y}_{v}\right)}\right\}$ for some small $\tau>0$. We used 
$\tau=0.01$. The true curve clearly displays important interesting information. The benefit of treatment $A$ is essentially restricted to those above the median value for $Y$. However, variability in the nonparametric estimate of $d(v)$ masks the result and the parametric estimator is biased to the point of being misleading when the model for $\operatorname{logit} P\left[D=1 \mid Y=y_{v}, T\right]$ is misspecified as linear in $v$. Therefore, as mentioned earlier, estimating $\theta(v)$ rather than $d(v)$ is a more tenable task. Moreover, the bottom line of how to set the treatment assignment policy using a threshold criterion derives easily from the SI curve.

\section{Discussion}

We have proposed calculating the SI function, $\theta(v)=\mathrm{P}\left[D=1 \mid\left(Y>y_{v}, T=1\right)\right.$ or $(Y<$ $\left.\left.y_{v}, T=0\right)\right]$, to evaluate the impact of a treatment selection policy based on the criterion " $Y>y_{v} . "$ The curve shows the range of operating characteristics that can be achieved across different thresholds $y_{v}$, where operating characteristics are defined in terms of the overall population response rate, i.e., $\theta(v)$, and the proportion of the population assigned to treatment $B$, i.e., $v$. We have mentioned that there are some similarities with receiver operative characteristic (ROC) curves used to evaluate diagnostic tests (Pepe 2000), but the purpose and application is very different. A potentially important use of the curve is for comparing different markers in regards to their capacities for selecting treatments. We have not addressed formal methods for making comparisons in this paper but propose to develop methods for such purposes in the future.

This paper has focused on a binary outcome variable $D$ and a continuous selection marker $Y$. If the potential response $D^{0}$ and $D^{1}$ were continuous, then the SI function could be defined 
in terms of expectations:

$$
\begin{aligned}
\theta(v) & =E\left(D \mid\left(T=1, Y>y_{v}\right) \text { or }\left(T=0, Y<y_{v}\right)\right) \\
& =(1-v) E\left(D^{1} \mid Y>y_{v}\right)+v E\left(D^{0} \mid Y<y_{v}\right)
\end{aligned}
$$

and estimation methods analogous to those described in this paper, $\widehat{\theta}_{n p}(v)$ and $\widetilde{\theta}_{p}(v)$, could be pursued. Alternative definitions for $\theta(v)$ are also possible of course, using quantiles instead of expectations, for example. If the selection marker $Y$ is discrete, the SI curve is a discrete function. Again procedures described here already can be used to estimate $\theta(v)$. Comparisons between markers are less straightforward with discrete $Y$ however than they are for continuous $Y$. In particular, decision criteria based on $F_{1}\left(Y_{1}\right)>v$ and $F_{2}\left(Y_{2}\right)>v$, may not yield comparable proportions of subjects assigned to treatment $A$, where subscripts here denote markers 1 and 2 respectively.

We have noted that there are several important assumptions that must be made about the clinical trial for valid estimation of the population response rate $\theta(v)$ from it. These are listed as items (i), (ii) and (iii) in Section 3. Item (i) is violated if subjects enrolling in the trial are not a simple random sample from the population. Although eligibility criteria attempt to define the population, this may not succeed, particularly if subjects self select to the study. This limits the generalizability of trial results to the "eligible" population and therefore applies to our problem of estimating the population SI curve too. If characteristics of the trial population simply differs from the population of interest in the distribution of some known covariates, one could reweight the data according to the population distribution and proceed with estimation. In the CTS study, one of the 3 study clinics is the Seattle Veterans Affairs Medical Center. Therefore, males will likely be over represented in the study. A reweighting scheme could be employed to adjust for this. The condition item (ii) 
relates to the response of patients observed in the trial being reflective of their response to treatment offered in the population setting. If patient care or compliance in the trial differs from what occurs in the population then this condition will not be met. Again, this is a general issue regarding the interpretation of trial results for populations and highlights the need to conduct trials in ways that reflect general practice. The final requirement, item (iii), is that in the trial, $Y$ is not used to select treatment or to dictate any aspect of patient care. This is ensured in the CTS study by having the MRNI performed by personnel not involved in patient care and storing the images until after the patient has completed the 12 month study.

Although the SI curve is one perspective from which to evaluate treatment selection markers, selection markers could be evaluated using other summary statistical measures too. For example, a utility function that incorporates notions of cost and benefit might be employed. A referee suggests the criterion to assign treatment $A$ if the probability of benefit on $A$ given $Y>y$ exceeds that on $B$ by a critical amount $\delta$, i.e., if $d^{+}(v)>\delta$. We hope that this paper will at a minimum stimulate statisticians to think critically and in new ways about the general problem of how to evaluate treatment selection markers.

\section{ACKNOWLEDGEMENTS}

This research was supported by NIH grants U01 CA86368 (Pepe) and M01-RR-00037 (Pepe) and U01-AI46702 (Song). The authors thank Noelle Noble and Gary Longton for their help with preparing the manuscript and Eugene Huang and Patrick Heagerty for useful discussions. 


\section{RÉSUMÉ}

\section{REFERENCES}

Andersen, P.K. and Gill, R.D. (1982). Cox's Regression Model for Counting Processes: A Large Sample Study. The Annals of Statistics 10, 1100-1120.

Byar D.P. (1985) Assessing apparent treatment-covariate interactions in randomized clinical trials. Statistics in Medicine 4:255-263.

Elmer-Dewitt, P., Lemonick, M., Park, A., and Nash, M. (2001). Medicine: the future of drugs. Time 157, 56-102.

Holland, P. (1986). Statistics and causal inference. Journal of the American Statistical Association $\mathbf{8 1}, 945-960$.

Pepe, M.S. (2000). Receiver operating characteristic methodology. Journal of the American StatisticalAssociation 95, 308-311.

Pepe, M.S. (2003). The Statistical Evaluation of Medical Tests for Classification and Prediction. Oxford University Press.

Rubin, D.B. (1974). Estimating causal effects of treatments in randomized and nonrandomized studies. Journal of Educational Psychology 66, 688-701.

Rubin, D.B. (1978). Bayesian inference for causal effects: The role of randomization. Annals of Statistics 6, 34-58.

Rubin, D.B. (1986). Statistics and causal inference: Comment: Which ifs have causal answers. Journal of the American Statistical Association 81, 961-962.

Serfling, R.J. (1967). Approximation Theorems of Mathematical Statistics. New York: Springer-Verlag. 
Van der Varrt (2000). Asymptotic Statistics. Cambridge: Cambridge University Press.

Van der Varrt, A.W. and Wellner, J.A. (2000). Weak Convergence and Empirical Processes. New York: Springer-Verlag. 


\section{Appendix A}

\section{Large sample properties of $\widehat{\theta}_{n p}(v)$}

Asymptotic distribution theory for $\widehat{\theta}_{n p}(v)$ follows from that of the component empirical processes. The empirical process $\sqrt{n}\left\{\left(F_{n}, G_{1 n}^{D}, G_{1 n}, G_{2 n}^{D}, G_{2 n}\right)-\left(F, G_{1}^{D}, G_{1}, G_{2}^{D}, G_{2}\right)\right\}$ converges to a tight, zero-mean Gaussian process $\mathbb{H}=\left(\mathbb{F}, \mathbb{G}_{1}^{D}, \mathbb{G}_{1}, \mathbb{G}_{2}^{D}, \mathbb{G}_{2}\right)$ with covariance structure

$$
\Sigma(y, x)=\operatorname{cov}\{\mathbb{H}(y), \mathbb{H}(x)\}=\operatorname{cov}\{h(y), h(x)\}
$$

where

$$
h(y)=\left(\begin{array}{c}
I(Y \leq y)-F(y) \\
I(Y>y, T=1, D=1)-G_{1}^{D}(y) \\
I(Y>y, T=1)-G_{1}(y) \\
I(Y \leq y, T=0, D=1)-G_{2}^{D}(y) \\
I(Y \leq y, T=0)-G_{2}(y)
\end{array}\right) .
$$

With some straightforward algebra, letting $\Delta G(y, x)=G(y)-G(x)$, we can show

$$
\begin{aligned}
& \Sigma(y, x)= \\
& \left\{\begin{array}{ccccc}
F(y \wedge x) & I(y>x) \Delta G_{1}^{D}(y, x) & I(y>x) \Delta G_{1}(y, x) & G_{2}^{D}(y \wedge x) & G_{2}(y \wedge x) \\
I(x>y) \Delta G_{1}^{D}(x, y) & G_{1}^{D}(y \vee x) & G_{1}^{D}(y \vee x) & 0 & 0 \\
I(x>y) \Delta G_{1}(x, y) & G_{1}^{D}(y \vee x) & G_{1}(y \vee x) & 0 & 0 \\
G_{2}^{D}(y \wedge x) & 0 & 0 & G_{2}^{D}(y \wedge x) & G_{2}^{D}(y \wedge x) \\
G_{2}(y \wedge x) & 0 & 0 & G_{2}^{D}(y \wedge x) & G_{2}(y \wedge x)
\end{array}\right\} \\
& -Q(y) Q(x),
\end{aligned}
$$

where $Q(y)=\left(F(y), G_{1}^{D}(y), G_{1}(y), G_{2}^{D}(y), G_{2}(y)\right)^{T}$. As a functional of $\left(F, G_{1}^{D}, G_{1}, G_{2}^{D}, G_{2}\right)$, $\theta$ is Hadamand-differentiable and the derivative can be derived by the chain rule (van der Vaart and Wellner 2000, §3.9). Let $f(y)=\frac{d F(y)}{d(y)}$. For $v \in[a, b](0<a<b<1)$ such that $f(y)>0$ on the interval $\left[F^{-1}(a)-\epsilon, F^{-1}(b)+\epsilon\right]$ for some positive $\epsilon$, using the functional 
delta method, $\sqrt{n}\left\{\widehat{\theta}_{n p}(v)-\theta(v)\right\}$ converges to a Gaussian process $\mathbb{Z}$ where,

$$
\begin{aligned}
\mathbb{Z}(v)= & (1-v)\left[\frac{\mathbb{G}_{1}^{D}\left(y_{v}\right)-g_{1}^{D}\left(y_{v}\right) \mathbb{F}\left(y_{v}\right) / f\left(y_{v}\right)}{G_{1}\left(y_{v}\right)}-\frac{G_{1}^{D}\left(y_{v}\right)\left\{\mathbb{G}_{1}\left(y_{v}\right)-g_{1}\left(y_{v}\right) \mathbb{F}\left(y_{v}\right) / f\left(y_{v}\right)\right\}}{G_{1}^{2}\left(y_{v}\right)}\right] \\
& +v\left[\frac{\mathbb{G}_{2}^{D}\left(y_{v}\right)-g_{2}^{D}\left(y_{v}\right) \mathbb{F}\left(y_{v}\right) / f\left(y_{v}\right)}{G_{2}\left(y_{v}\right)}-\frac{G_{2}^{D}\left(y_{v}\right)\left\{\mathbb{G}_{2}\left(y_{v}\right)-g_{2}\left(y_{v}\right) \mathbb{F}\left(y_{v}\right) / f\left(y_{v}\right)\right\}}{G_{2}^{2}\left(y_{v}\right)}\right] \\
= & A^{T}(v) \mathbb{H}\left(y_{v}\right),
\end{aligned}
$$

where $g_{j}^{D}(y)=\frac{d G_{j}^{D}(y)}{d y}, g_{j}(y)=\frac{d G_{j}(y)}{d y}, j=1,2$,

$$
A(v)=\left(\begin{array}{c}
-\left\{\frac{(1-v) g_{1}^{D}\left(y_{v}\right)}{G_{1}\left(y_{v}\right)}-\frac{(1-v) G_{1}^{D}\left(y_{v}\right) g_{1}(v)}{G_{1}^{2}\left(y_{v}\right)}+\frac{v g_{2}^{D}\left(y_{v}\right)}{G_{2}\left(y_{v}\right)}-\frac{v G_{2}^{D}\left(y_{v}\right) g_{2}(v)}{G_{2}^{2}\left(y_{v}\right)}\right\} \frac{1}{f\left(y_{v}\right)} \\
\frac{1-v}{G_{1}\left(y_{v}\right)} \\
-\frac{(1-v) G_{1}^{D}\left(y_{v}\right)}{G_{1}^{2}\left(y_{v}\right)} \\
\frac{v}{G_{2}\left(y_{v}\right)} \\
-\frac{v G_{2}^{D}\left(y_{v}\right)}{G_{2}^{2}\left(y_{v}\right)}
\end{array}\right),
$$

and $\operatorname{cov}(\mathbb{Z}(s), \mathbb{Z}(t))=A^{T}(s) \Sigma\left(y_{s}, y_{t}\right) A(t)$. The asymptotic variance for $\widehat{\theta}_{n p}(v)$ therefore is

$$
V_{n p}(v)=n^{-1} A^{T}(v) \Sigma\left(y_{v}, y_{v}\right) A(v)
$$

In applications, we can use the bootstrap method to calculate a confidence band for $\theta(v)$ for $v \in[a, b](0<a<b<1)$. This follows from theory for the bootstrap given in van der Vaart and Wellner $(2000, \S 3.9 .3)$. Let $Z_{i}=\left(D_{i}, Y_{i}, T_{i}\right), \Theta=\left(Z_{1}, \ldots, Z_{n}\right)$ be the observed data set and $\Theta_{B}=\left(Z_{1}(B), Z_{2}(B), \ldots, Z_{n}(B)\right)$ be the $B^{\text {th }}$ resampling bootstrap dataset, $B=1,2 \ldots, K$. Let $\widehat{\theta}_{n p}^{B}$ be the estimator based on $\Theta_{B}$. Then $\sup _{v \in[a, b]}\left|\widehat{\theta}_{n p}^{B}(v)-\widehat{\theta}_{n p}(v)\right|$ given $\Theta$ is asymptotically equivalent to $\sup _{v \in[a, b]}\left|\widehat{\theta}_{n p}(v)-\theta(v)\right|$. Let $c_{\alpha}$ be the $1-\alpha$ quantile of $\sup _{v \in[a, b]}\left|\widehat{\theta}_{n p}^{B}(v)-\widehat{\theta}_{n p}(v)\right|$, then a level $\alpha$ confidence band for $\theta(v)$ is $\left(\widehat{\theta}_{n p}^{B}(v)-c_{\alpha}, \widehat{\theta}_{n p}^{B}(v)+c_{\alpha}\right)$. It may be preferable to calculate confidence bands after a logit transformation. When $\theta(v)>0$ on $[a, b], \sqrt{n}\{\operatorname{logit}(\widehat{\theta})-\operatorname{logit}(\theta)\}$ converges to a Gaussian process by the delta method. Letting $\tilde{c}_{\alpha}$ be the $1-\alpha$ quantile of $\sup _{v \in[a, b]}\left|\operatorname{logit}\left\{\widehat{\theta}_{n p}^{B}(v)\right\}-\operatorname{logit}\left\{\widehat{\theta}_{n p}(v)\right\}\right|$, a level $\alpha$ confidence band for $\theta(v)$ is $\operatorname{logit}{ }^{-1}\left(\left\{\operatorname{logit}\left\{\widehat{\theta}_{n p}^{B}(v)\right\}-\widetilde{c}_{\alpha}, \operatorname{logit}\left\{\widehat{\theta}_{n p}^{B}(v)\right\}+\widetilde{c}_{\alpha}\right)\right.$. 


\section{Appendix B}

Large sample properties of $\widehat{\theta}_{p}(v)$ and $\widetilde{\theta}_{p}(v)$

Let $\alpha_{0}$ be the true value of $\alpha$. Since $\sqrt{n}\left(\hat{\alpha}-\alpha_{0}\right)$ is asymptotically normal with mean 0 and variance the inverse expected information denoted by $V_{\alpha}$, and $\partial \theta / \partial \alpha$ is bounded under the

assumption that $\int_{0}^{1}|R(u)| d u<\infty$, by the functional delta method, we have the following theorem.

Theorem 2. $\sqrt{n}\left\{\hat{\theta}_{p}(v)-\theta(v)\right\}$ converges to a Gaussian process $\mathbb{Z}_{p}$ with covariance structure

$$
\Lambda\left(v_{1}, v_{2} ; V_{\alpha}\right)=\operatorname{cov}\left\{\mathbb{Z}_{p}\left(v_{1}\right), \mathbb{Z}_{p}\left(v_{2}\right)\right\}=\frac{\partial \theta\left(v_{1} ; \alpha_{0}, F\right)}{\alpha^{T}} V_{\alpha}\left\{\frac{\partial \theta\left(v_{2} ; \alpha_{0}, F\right)}{\alpha^{T}}\right\}^{T}
$$

Now we consider $\widetilde{\theta}_{p}(v)$. First, we derive the large sample properties of $\tilde{\alpha}$. For technical reasons we restrict estimation of $\tilde{\alpha}$ to $F_{n}^{*}\left(Y_{i}\right) \in(a, b)$, a proper subset of $(0,1)$, and assume that $R(v, t)$ has uniformly continuous and bounded partial derivatives $R^{(1)}(v, t)$ in the interval $\left(a-\epsilon_{0}, b+\epsilon_{0}\right), 0<a<b<1$, for some positive $\epsilon_{0}$. If this fails at some interior points, we can restrict estimation to the union $\bigcup_{s=1}^{S}\left(a_{s}, b_{s}\right)$ where the condition does hold. Let $W_{i}=F\left(Y_{i}\right)$, $\hat{W}_{i}=F_{n}\left(Y_{i}\right), Z_{i}=\left(D_{i}, W_{i}, T_{i}\right)^{T}, \hat{Z}_{i}=\left(D_{i}, \hat{W}_{i}, T_{i}\right)^{T}$. The estimator $\tilde{\alpha}$ is the solution to the following estimating equations

$$
\tilde{U}(\alpha ; Z)=n^{-1} \sum_{i=1}^{n} I\left\{\hat{W}_{i} \in(a, b)\right\} \phi\left(\alpha ; Z_{i}\right)=0,
$$

where $\phi\left(\alpha ; Z_{i}\right)=\left[D_{i}-\frac{\exp \left\{\alpha^{T} R\left(W_{i}, T_{i}\right)\right\}}{1+\exp \left\{\alpha^{T} R\left(W_{i}, T_{i}\right)\right\}}\right] R\left(W_{i}, T_{i}\right)$.

1. Consistency of $\tilde{\alpha}$

First we show $\sqrt{n}\left\{F_{n}^{*}-F\right\}$ converges to a tight Gaussian process. Note $\sqrt{n}\left\{F_{n}^{*}-F\right\}=$ $\sqrt{n}\left\{F_{n}-F\right\}+\sqrt{n}\left\{F_{n}^{*}-F_{n}\right\}$. The empirical process $\sqrt{n}\left\{F_{n}-F\right\}$ converges to a tight 
Gaussian process, and

$$
\sup _{y \in \mathbb{R}}\left|\sqrt{n}\left\{F_{n}^{*}(y)-F_{n}(y)\right\}\right|=\frac{n^{1 / 2}}{n+1} \sup _{y \in \mathbb{R}}\left|F_{n}(y)\right| \leq \frac{n^{1 / 2}}{n+1}=o_{p}(1),
$$

since $\left|F_{n}(y) \leq 1\right|$. Hence the result follows.

Let $W_{i}=F\left(Y_{i}\right), Z_{i}=\left(D_{i}, W_{i}, T_{i}\right)^{T}$, and $\hat{Z}_{i}=\left(D_{i}, \hat{W}_{i}, T_{i}\right)^{T}$. Denote the true value of $\alpha$ by $\alpha_{0}$. The estimator $\tilde{\alpha}$ maximizes the concave function of $\alpha$

$$
l\left(\alpha ; \hat{Z}_{i}\right)=n^{-1} \sum_{i=1}^{n} I\left\{\hat{W}_{i} \in(a, b)\right\}\left(D_{i} R\left(\hat{W}_{i}, T_{i}\right)-\log \left[1+\exp \left\{\alpha^{T} R\left(\hat{W}_{i}, T_{i}\right)\right\}\right]\right) .
$$

By the Median Value Theorem,

$$
\begin{aligned}
& \left|l\left(\alpha ; \hat{Z}_{i}\right)-l\left(\alpha ; Z_{i}\right)\right| \\
= & \left|n^{-1} \sum_{i=1}^{n} I\left\{\hat{W}_{i} \in(a, b)\right\}\left[D_{i}-\frac{\exp \left\{\alpha^{T} \tilde{R}_{i}\right\}}{1+\exp \left\{\alpha^{T} \tilde{R}_{i}\right\}} \alpha\right]\left\{R\left(\hat{W}_{i}, T_{i}\right)-R\left(W_{i}, T_{i}\right)\right\}\right|,
\end{aligned}
$$

where $\tilde{R}_{i}$ is on the line segment between $R\left(\hat{W}_{i}, T_{i}\right)$ and $R\left(W_{i}, T_{i}\right)$. Since $\sup _{y \in \mathbb{R}} \mid F_{n}^{*}(y)-$ $F_{n}(y) \mid<\epsilon \rightarrow 0$, for any $\epsilon>0$, there exists $N_{\epsilon}$ such that $\sup _{y \in \mathbb{R}}\left|F_{n}^{*}(y)-F_{n}(y)\right|<\epsilon$ for $n>N_{\epsilon}$. Thus $\hat{W}_{i} \in(a, b)$ implies $W_{i} \in\left(a-\epsilon_{0}, a+\epsilon_{0}\right)$ and hence $I\left\{\hat{W}_{i} \in(a, b)\right\} \leq$ $I\left\{W_{i} \in\left(a-\epsilon_{0}, b+\epsilon_{0}\right)\right\}$ for $n>N_{\epsilon_{0}}$. Replacing the right side of (B.1) by its upper bound, we have, for $n>N_{\epsilon_{0}}$,

$$
\begin{aligned}
\left|l\left(\alpha ; \hat{Z}_{i}\right)-l\left(\alpha ; Z_{i}\right)\right| & \leq(1+\|\alpha\|) \sup _{i}\left[I\left\{W_{i} \in\left(a-\epsilon_{0}, b+\epsilon_{0}\right)\right\}\left|R\left(\hat{W}_{i}, T_{i}\right)-R\left(W_{i}, T_{i}\right)\right|\right] \\
& \leq(1+\|\alpha\|) \max _{t \in\{0,1\}} \sup _{F(y) \in\left(a-\epsilon_{0}, b+\epsilon_{0}\right)}\left|n^{-1} \sqrt{n}\left[R\left\{F_{n}^{*}(y), t\right\}-R\{F(y), t\}\right]\right| \\
& =o_{p}(1),
\end{aligned}
$$

The last equality follows from that $\sqrt{n}\left[R\left\{F_{n}^{*}, t\right\}-R\{F, t\}\right]$ converges to a Gaussian process by delta method under the assumption (I) on $v \in\left(a-\epsilon_{0}, b+\epsilon_{0}\right)$, and hence $n^{-1} \sqrt{n}\left[R\left\{F_{n}^{*}, t\right\}-\right.$ 
$R\{F, t\}]$ converges to 0 in distribution by Slutsky theorem for $t=0,1$. Coupled with $l\left(\alpha ; Z_{i}\right) \stackrel{P}{\rightarrow} \eta(\alpha)=\mathrm{E}\left[I\left\{W_{i} \in(a, b)\right\}\left(D_{i} R\left(W_{i}, T_{i}\right)-\log \left[1+\exp \left\{\alpha^{T} R\left(W_{i}, T_{i}\right)\right\}\right]\right)\right]$ (central limit theorem), we have $l\left(\alpha ; \hat{Z}_{i}\right) \stackrel{P}{\rightarrow} \eta(\alpha)$. By Corollary II.2 of Andersen and Gill (1982), the consistency of $\tilde{\alpha}$ follows.

2. Asymptotic normality of $\tilde{\alpha}$

By a Taylor series expansion,

$$
\begin{aligned}
& \sqrt{n}\left\{\tilde{U}\left(\alpha_{0} ; \hat{Z}\right)-\tilde{U}\left(\alpha_{0} ; Z\right)\right\} \\
= & n^{-1 / 2} \sum_{i=1}^{n} I\left\{\hat{W}_{i} \in(a, b)\right\} \lambda_{1}\left(\alpha_{0} ; Z_{i}\right)\left\{R\left(\hat{W}_{i}, T_{i}\right)-R\left(W_{i}, T_{i}\right)\right\} \\
& +Q\left(\alpha_{0} ; Z_{i}, \hat{Z} i\right)
\end{aligned}
$$

where

$$
\lambda_{1}\left(\alpha_{0} ; Z_{i}\right)=-\frac{\exp \left\{\alpha^{T} R\left(W_{i}, T_{i}\right)\right\}}{\left[1+\exp \left\{\alpha^{T} R\left(W_{i}, T_{i}\right)\right\}\right]^{2}} R\left(W_{i}, T_{i}\right) \alpha^{T}+\left\{D_{i}-\frac{\exp \left\{\alpha^{T} R\left(W_{i}, T_{i}\right)\right\}}{1+\exp \left\{\alpha^{T} R\left(W_{i}, T_{i}\right)\right\}}\right\} I_{q},
$$

and $I_{q}$ is a $q \times q$ identity matrix, and $Q\left(\alpha_{0} ; Z_{i}, \hat{Z}_{i}\right)$ is a $q$ dimensional vector with the $m^{\text {th }}$ element equal to

$$
\begin{aligned}
& Q_{m}\left(\alpha_{0} ; Z_{i}, \hat{Z}_{i}\right)= n^{-1} \sum_{i=1}^{n} I\left\{\hat{W}_{i} \in(a, b)\right\}\left\{R\left(\hat{W}_{i}, T_{i}\right)-R\left(W_{i}, T_{i}\right)\right\}^{T} \\
& \times \lambda_{2}\left(\alpha_{0} ; \tilde{R}_{i}^{*}\right)\left[\sqrt{n}\left\{R\left(\hat{W}_{i}, T_{i}\right)-R\left(W_{i}, T_{i}\right)\right\}\right], \\
& \lambda_{2}\left(\alpha_{0} ; \tilde{R}_{i}^{*}\right)=\frac{1}{2}\left[-\frac{\exp \left\{\alpha^{T} \tilde{R}_{i}^{*}\right\}}{\left[1+\exp \left\{\alpha^{T} \tilde{R}_{i}^{*}\right\}\right]^{2}} \tilde{R}_{i m}^{*} \alpha \alpha^{T}+\frac{2\left\{\exp \left(\alpha^{T} \tilde{R}_{i}^{*}\right)\right\}^{2}}{\left\{1+\exp \left(\alpha^{T} \tilde{R}_{i}^{*}\right)\right\}^{3}} \tilde{R}_{i m}^{*} \alpha \alpha^{T}\right. \\
&\left.-\frac{2 \exp \left\{\alpha^{T} \tilde{R}_{i}^{*}\right\}}{\left\{1+\exp \left\{\alpha^{T} \tilde{R}_{i}^{*}\right\}\right\}^{2}} E\right],
\end{aligned}
$$

$\tilde{R}_{i}^{*}$ is on the line segment between $R\left(\hat{W}_{i}, T_{i}\right)$ and $R\left(W_{i}, T_{i}\right), \tilde{R}_{i m}^{*}$ is the $m^{\text {th }}$ element of $\tilde{R}_{i}^{*}$, $E$ is a $q \times q$ matrix with the $m^{\text {th }}$ column equal to $\alpha$ and all other columns equal to 0 . Now 
we show $Q_{m}\left(\alpha_{0} ; Z_{i}, \hat{Z}_{i}\right)=o_{p}(1)$. With simple algebra, we can show that there exists a $q \times q$ matrix $M$ with finite positive elements such that

$$
\sup _{\{z=(d, w, t): d, t=0,1, w \in(a-\epsilon, b+\epsilon)\}}\left|\lambda_{j l m}\left(\alpha_{0} ; z\right)\right|<M
$$

for $j=1,2$, where " $<$ " holds elementwisely. Hence

$$
\begin{aligned}
Q_{m}\left(\alpha_{0} ; Z_{i}, \hat{Z} i\right) \leq \sup _{i}\left(I\left\{\hat{W}_{i} \in(a, b)\right\}\left|\left\{R\left(\hat{W}_{i}, T_{i}\right)-R\left(W_{i}, T_{i}\right)\right\}^{T}\right|\right. \\
\left.\quad \times M\left|\sqrt{n}\left\{R\left(\hat{W}_{i}, T_{i}\right)-R\left(W_{i}, T_{i}\right)\right\}\right|\right) \\
\leq \sup _{i}\left(I\left\{W_{i} \in\left(a-\epsilon_{0}, b+\epsilon_{0}\right)\right\}\left|\left[R\left\{F_{n}^{*}\left(Y_{i}\right), T_{i}\right\}-R\left\{F\left(Y_{i}\right), T_{i}\right\}\right]^{T}\right|\right. \\
\left.\quad \times M\left|\sqrt{n}\left[R\left\{F_{n}^{*}\left(Y_{i}\right), T_{i}\right\}-R\left\{F\left(Y_{i}\right), T_{i}\right\}\right]\right|\right) \\
\leq \max _{t \in\{0,1\}} \sup _{F(y) \in\left(a-\epsilon_{0}, b+\epsilon_{0}\right)}\left|\left[R\left\{F_{n}^{*}(y), t\right\}-R\{F(y), t\}\right]^{T}\right| \\
\quad \times M\left|\sqrt{n}\left[R\left\{F_{n}^{*}(y), t\right\}-R\{F(y), t\}^{T}\right]\right| \\
=o_{p}(1) .
\end{aligned}
$$

The last equality follows from that $\sqrt{n}\left[R\left\{F_{n}^{*}, t\right\}-R\{F, t\}\right]$ converges to a Gaussian process on $\left(a-\epsilon_{0}, b+\epsilon_{0}\right)$ by the delta method and hence $\left[R\left\{F_{n}^{*}, t\right\}-R\{F, t\}\right]$ converges in probability to 0 and then use the Slutsky theorem. Hence $(B .3)=o_{p}(1)$. Next, by adding and subtracting a term, (B.2) can be expressed as

$$
\begin{aligned}
& n^{-1 / 2} \sum_{i=1}^{n} I\left\{\hat{W}_{i} \in(a, b)\right\} \lambda_{1}\left(\alpha_{0} ; Z_{i}\right) R^{(1)}\left(W_{i}, T_{i}\right)\left(\hat{W}_{i}-W_{i}\right) \\
& +n^{-1} \sum_{i=1}^{n} I\left\{\hat{W}_{i} \in(a, b)\right\} \lambda_{1}\left(\alpha_{0} ; Z_{i}\right) \sqrt{n}\left[\left\{R\left(\hat{W}_{i}\right)-R\left(W_{i}\right)\right\}-R^{(1)}\left(W_{i}, T_{i}\right)\left(\hat{W}_{i}-W_{i}\right)\right] .
\end{aligned}
$$


(B.5) is bounded by

$$
\begin{aligned}
& M \sup _{i} I\left\{W_{i} \in\left(a-\epsilon_{0}, b+\epsilon_{0}\right)\right\} \\
& \quad \times\left|\sqrt{n}\left(\left[R\left\{F_{n}^{*}\left(Y_{i}\right), T_{i}\right\}-R\left\{F\left(Y_{i}\right), T_{i}\right\}\right]-R^{(1)}\left\{F\left(Y_{i}\right), T_{i}\right\}\left\{F_{n}^{*}\left(Y_{i}\right)-F\left(Y_{i}\right)\right\}\right)\right| \\
& \leq M \max _{\substack{t \in\{0,1\} \\
s \in\{1, \ldots, S\}}} \sup _{(y) \in\left(a-\epsilon_{0}, b+\epsilon_{0}\right)} \mid \sqrt{n}\left(\left[R\left\{F_{n}^{*}(y), t\right\}-R\{F(y), t\}\right]\right. \\
& \left.\quad-R^{(1)}\left\{F\left(Y_{i}\right), T_{i}\right\}\left\{F_{n}^{*}(y)-F(y)\right\}\right) \mid \\
& =M \max _{t \in\{0,1\}} \sup _{F(y) \in\left(a-\epsilon_{0}, b+\epsilon_{0}\right)} \mid \sqrt{n}\left(\left[R\left\{F_{n}^{*}(y), t\right\}-R^{(1)}\{F(y), t\} F_{n}^{*}(y)\right]\right. \\
& \left.\quad-\left[R\{F(y), t\}-R^{(1)}\{F(y), t\}\left\{F_{n}^{*}(y)-F(y)\right\}\right]\right) \mid \\
& =o_{p}(1) .
\end{aligned}
$$

The last equality follows from that the process

$$
\sqrt{n}\left(\left[R\left\{F_{n}^{*}, t\right\}-R^{(1)}(F, t) F_{n}^{*}\right]-\left[R\{F, t\}-R^{(1)}(F, t) F\right]\right)
$$

converges to 0 on $\left(a-\epsilon_{0}, b+\epsilon_{0}\right)$ by the delta method. Now we show (B.4) is equal to

$$
n^{-1 / 2} \sum_{i=1}^{n} I\left\{W_{i} \in(a, b)\right\} \lambda_{1}\left(\alpha_{0} ; Z_{i}\right) R^{(1)}\left(W_{i}, T_{i}\right)\left(\hat{W}_{i}-W_{i}\right)+o_{p}(1) .
$$


For any $\epsilon<\epsilon_{0}$ and $n>N_{\epsilon}$,

$$
\begin{aligned}
& \left|n^{-1 / 2} \sum_{i=1}^{n}\left[I\left\{\hat{W}_{i} \in(a, b)\right\}-I\left\{W_{i} \in(a, b)\right\}\right] \lambda_{1}\left(\alpha_{0} ; Z_{i}\right) R^{(1)}\left(W_{i}, T_{i}\right)\left(\hat{W}_{i}-W_{i}\right)\right| \\
\leq & n^{-1 / 2} \sum_{i=1}^{n}\left[I\left\{\hat{W}_{i} \in(a, b)\right\} I\left\{W_{i} \notin(a, b)\right\}+I\left\{\hat{W}_{i} \notin(a, b)\right\} I\left\{W_{i} \in(a, b)\right\}\right] \\
& \times\left|I\left\{W_{i} \in(a-\epsilon, b+\epsilon)\right\} \lambda_{1}\left(\alpha_{0} ; Z_{i}\right) R^{(1)}\left(W_{i}, T_{i}\right)\left(\hat{W}_{i}-W_{i}\right)\right| \\
\leq & n^{-1} \sum_{i=1}^{n}\left[I\left\{\hat{W}_{i} \in(a, b)\right\} I\left\{W_{i} \notin(a, b)\right\}+I\left\{\hat{W}_{i} \notin(a, b)\right\} I\left\{W_{i} \in(a, b)\right\}\right] \\
& \times \sup _{i}\left|I\left\{W_{i} \in(a-\epsilon, b+\epsilon)\right\} \lambda_{1}\left(\alpha_{0} ; Z_{i}\right) R^{(1)}\left(W_{i}, T_{i}\right) \sqrt{n}\left(\hat{W}_{i}-W_{i}\right)\right| \\
\leq \quad & n^{-1} \sum_{i=1}^{n}\left[I\left\{W_{i} \in(a+\epsilon, b-\epsilon)\right\} I\left\{W_{i} \notin(a, b)\right\}\right. \\
& \left.+I\left\{W_{i} \notin(a-\epsilon, b+\epsilon)\right\} I\left\{W_{i} \in(a, b)\right\}\right] \\
& \times M \max _{t \in\{0,1\}} \sup _{w \in[a-\epsilon, b+\epsilon]}\left|R^{(1)}(w, t)\right| \sup _{y \in \mathbb{R}}\left|\sqrt{n}\left\{F_{n}^{*}(y)-F(y)\right\}\right| \\
\leq & n^{-1} \sum_{i=1}^{n}\left[I\left\{W_{i} \in[a-\epsilon, a] \cup[b, b+\epsilon]\right\}+I\left\{W_{i} \in(a, a+\epsilon) \cup[b-\epsilon, b]\right\}\right] \\
& \times M \max _{t \in\{0,1\}} \sup _{w \in[a-\epsilon, b+\epsilon]}\left|R^{(1)}(w, t)\right| \sup _{y \in \mathbb{R}}\left|\sqrt{n}\left\{F_{n}^{*}(y)-F(y)\right\}\right| \\
\leq & \sum_{i=1}^{n} I\left\{W_{i} \in[a-\epsilon, a+\epsilon] \cup[b-\epsilon, b+\epsilon]\right\} \\
\leq & \max _{t \in\{0,1\}} \sup _{w \in(a-\epsilon, b+\epsilon)}\left|R^{(1)}(w, t)\right| \sup _{y \in \mathbb{R}}\left|\sqrt{n}\left\{F_{n}^{*}(y)-F(y)\right\}\right|
\end{aligned}
$$

By the strong law of large number,

$$
n^{-1} \sum_{i=1}^{n} I\left\{W_{i} \in[a-\epsilon, a+\epsilon] \cup[b-\epsilon, b+\epsilon]\right\} \stackrel{a . s .}{\rightarrow} \operatorname{Pr}\left(W_{i} \in[a-\epsilon, a+\epsilon] \cup[b-\epsilon, b+\epsilon]\right) \leq 4 \epsilon .
$$

And

$$
\sup _{w \in\left(a-\epsilon_{0}, b+\epsilon_{0}\right)}\left|R^{(1)}(w, t)\right|<M^{*} \text { for some } M^{*}(q \times 1) .
$$


By DKW inequality (van der Vaart, 2000, P268),

$$
\operatorname{Pr}\left\{\sup _{y \in \mathbb{R}}\left|\sqrt{n}\left\{F_{n}^{*}(y)-F(y)\right\}\right|>\sqrt{\log (2 / \epsilon)}\right\}<\epsilon
$$

Combining (B.7-B.10)), for $n>\max \left(N_{\epsilon}\right)$,

$$
\begin{aligned}
& \operatorname{Pr}\left\{\left|n^{-1 / 2} \sum_{i=1}^{n}\left[I\left\{\hat{W}_{i} \in(a, b)\right\}-I\left\{W_{i} \in(a, b)\right\}\right] \lambda_{1}\left(\alpha_{0} ; Z_{i}\right) R^{(1)}\left(W_{i}, T_{i}\right)\left(\hat{W}_{i}-W_{i}\right)\right|\right. \\
> & \left.8 S M M^{*} \epsilon \sqrt{\log (2 / \epsilon)}\right\}<\epsilon .
\end{aligned}
$$

Hence

$$
n^{-1 / 2} \sum_{i=1}^{n}\left[I\left\{\hat{W}_{i} \in(a, b)\right\}-I\left\{W_{i} \in(a, b)\right\}\right] \lambda_{1}\left(\alpha_{0} ; Z_{i}\right) R^{(1)}\left(W_{i}, T_{i}\right)\left(\hat{W}_{i}-W_{i}\right)=o_{p}(1),
$$

and (B.6) holds. Therefore

$\sqrt{n}\left\{\tilde{U}\left(\alpha_{0} ; \hat{Z}\right)-\tilde{U}\left(\alpha_{0} ; Z\right)\right\}=n^{-1 / 2} \sum_{i=1}^{n} I\left\{W_{i} \in(a, b)\right\} \lambda_{1}\left(\alpha_{0} ; Z_{i}\right) R^{(1)}\left(W_{i}, T_{i}\right)\left(\hat{W}_{i}-W_{i}\right)+o_{p}(1)$.

Now substituting $\hat{W}_{i}=n^{-1} \sum_{j=1}^{n} I\left(Y_{j} \leq Y_{i}\right)$ in (B.11), we have

$$
\begin{aligned}
& \sqrt{n}\left\{\tilde{U}\left(\alpha_{0} ; \hat{Z}\right)-\tilde{U}\left(\alpha_{0} ; Z\right)\right\} \\
= & n^{-3 / 2} \sum_{i=1}^{n} \sum_{j=1}^{n} I\left\{W_{i} \in(a, b)\right\} \lambda_{1}\left(\alpha_{0} ; Z_{i}\right) R^{(1)}\left\{F\left(Y_{i}\right), T_{i}\right\}\left\{I\left(Y_{j} \leq Y_{i}\right)-F\left(Y_{i}\right)\right\}+o_{p}(1) \\
= & \frac{1}{2} \sqrt{n}\left\{\frac{1}{n^{2}} \sum_{i=1}^{n} \sum_{j=1}^{n} q\left(\alpha_{0} ; Z_{i}, Z_{j}\right)\right\}+o_{p}(1),
\end{aligned}
$$

where

$$
\begin{aligned}
q\left(\alpha_{0} ; Z_{i}, Z_{j}\right)= & I\left\{W_{i} \in(a, b)\right\} \lambda_{1}\left(\alpha_{0} ; Z_{i}\right) R^{(1)}\left\{F\left(Y_{i}\right), T_{i}\right\}\left\{I\left(Y_{j} \leq Y_{i}\right)-F\left(Y_{i}\right)\right\} \\
& +I\left\{W_{j} \in(a, b)\right\} \lambda_{1}\left(\alpha_{0} ; Z_{j}\right) R^{(1)}\left\{F\left(Y_{j}\right), T_{j}\right\}\left\{I\left(Y_{i} \leq Y_{j}\right)-F\left(Y_{j}\right)\right\}
\end{aligned}
$$


Note

$$
\xi=\frac{1}{n^{2}} \sum_{i=1}^{n} \sum_{j=1}^{n} q\left(\alpha_{0} ; Z_{i}, Z_{j}\right)
$$

is a $\mathrm{V}$-statistic with mean 0 . It is easy to show $\xi$ has finite variance. Hence $\sqrt{n} \xi$ has the same asymptotic distribution as $\sqrt{n} \xi^{*}$ (Serfling, 1967, §5.7.3), where

$$
\xi^{*}=\frac{1}{\left(\begin{array}{l}
n \\
2
\end{array}\right)} \sum_{i=1}^{n} \sum_{i<j}^{n} q\left(\alpha_{0} ; Z_{i}, Z_{j}\right)
$$

is the corresponding U-statistic. And $\sqrt{n} \xi^{*}$ is equivalent to (van der Vaart, 2000, §12.1)

$$
2 n^{-1 / 2} \sum_{i=1}^{n} q_{1}\left(\alpha_{0} ; Y_{i}\right)+o_{p}(1)
$$

where

$$
\begin{aligned}
& q_{1}\left(\alpha_{0} ; y\right)=\mathrm{E}\left\{q\left(\alpha_{0} ; z, Z_{2}\right)\right\} \\
& =\mathrm{E}\left[I\left\{W_{2} \in(a, b)\right\} \lambda_{1}\left(\alpha_{0} ; Z_{2}\right) R^{(1)}\left(W_{2}, T_{2}\right)\left\{I\left(y \leq Y_{2}\right)-F\left(Y_{2}\right)\right\}\right] \\
& =-\mathrm{E}\left[I\left\{W_{2} \in(a, b)\right\} \frac{\exp \left\{\alpha^{T} R\left(W_{2}, T_{2}\right)\right\}}{\left[1+\exp \left\{\alpha^{T} R\left(W_{2}, T_{2}\right)\right\}\right]^{2}} R\left(W_{2}, T_{2}\right) \alpha^{T} R^{(1)}\left(W_{2}, T_{2}\right)\right. \\
& \left.\quad \times\left\{I\left(y \leq Y_{2}\right)-F\left(Y_{2}\right)\right\}\right],
\end{aligned}
$$

and $z=(d, y, t)^{T}$, with the last two equalities in (B.12) follow from conditional expectation arguments. Hence

$$
\begin{aligned}
\sqrt{n} \tilde{U}\left(\alpha_{0} ; \hat{Z}\right) & =\sqrt{n} \tilde{U}\left(\alpha_{0} ; Z\right)+n^{-1 / 2} \sum_{i=1}^{n} q_{1}\left(\alpha_{0} ; Y_{i}\right)+o_{p}(1) \\
& =n^{-1 / 2} \sum_{i=1}^{n}\left[I\left\{W_{i} \in(a, b)\right\} \phi\left(\alpha_{0} ; Z_{i}\right)+q_{1}\left(\alpha_{0} ; Y_{i}\right)\right]+o_{p}(1) .
\end{aligned}
$$

By the central limit theorem, $\sqrt{n} \tilde{U}\left(\alpha_{0} ; \hat{Y}\right)$ is asymptotically normal with mean 0 and variance

$$
\begin{aligned}
C & =\operatorname{var}\left[I\left\{W_{i} \in(a, b)\right\} \phi\left(\alpha_{0} ; Z_{i}\right)+q_{1}\left(\alpha_{0} ; Y_{i}\right)\right] \\
& =\operatorname{var}\left[I\left\{W_{i} \in(a, b)\right\} \phi_{i}\left(\alpha_{0} ; Z_{i}\right)\right]+\operatorname{var}\left\{q_{1}\left(\alpha_{0} ; Y_{i}\right)\right\}
\end{aligned}
$$


The last equation follows from

$$
\begin{aligned}
& \operatorname{cov}\left[I\left\{W_{i} \in(a, b)\right\} \phi\left(\alpha_{0} ; Z_{i}\right), q_{1}\left(\alpha_{0} ; Y_{i}\right)\right] \\
= & \mathrm{E}\left[I\left\{W_{i} \in(a, b)\right\} \phi\left(\alpha_{0} ; Z_{i}\right) q_{1}\left(\alpha_{0} ; Y_{i}\right)^{T}\right] \\
= & \mathrm{E}\left(\mathrm{E}\left[I\left\{W_{i} \in(a, b)\right\} \phi\left(\alpha_{0} ; Z_{i}\right) q_{1}\left(\alpha_{0} ; Y_{i}\right)^{T} \mid Y_{i}, T_{i}\right]\right) \\
= & \mathrm{E}\left[I\left\{W_{i} \in(a, b)\right\} \mathrm{E}\left\{\phi\left(\alpha_{0} ; Z_{i}\right) \mid Y_{i}, T_{i}\right\} q_{1}\left(\alpha_{0} ; Y_{i}\right)^{T}\right] \\
= & 0 .
\end{aligned}
$$

Now by another Taylor series expansion,

$$
0=\sqrt{n} \tilde{U}(\tilde{\alpha} ; \hat{Z})=\sqrt{n} \tilde{U}\left(\alpha_{0} ; \hat{Z}\right)+\hat{\Gamma}\left(\alpha^{*} ; \hat{Z}\right) \sqrt{n}\left(\tilde{\alpha}-\alpha_{0}\right)
$$

where $\alpha^{*}$ is on the line segment between $\alpha$ and $\alpha_{0}$, and

$\hat{\Gamma}(\alpha ; Z)=\frac{\partial \tilde{U}(\alpha ; Z)}{\partial \alpha}=-n^{-1} \sum_{i=1}^{n} I\left\{W_{i} \in(a, b)\right\} \frac{\exp \left\{\alpha^{T} R\left(W_{i}, T_{i}\right)\right\}}{\left[1+\exp \left\{\alpha^{T} R\left(W_{i}, T_{i}\right)\right\}\right]^{2}} R\left(W_{i}, T_{i}\right) R\left(W_{i}, T_{i}\right)^{T}$

Let $\Gamma(\alpha)=\mathrm{E}\left\{\frac{\partial \tilde{U}(\alpha ; Z)}{\partial \alpha^{T}}\right\}$. Using similar arguments as those for proving $\left|l\left(\alpha ; \hat{Z}_{i}\right)-l\left(\alpha ; Z_{i}\right)\right|=$ $o_{p}(1)$, we have $\sup _{\alpha}|\hat{\Gamma}(\alpha ; \hat{Z})-\hat{\Gamma}(\alpha ; Z)|=o_{p}(1)$. Also $\left|\hat{\Gamma}(\alpha ; Z)-\hat{\Gamma}\left(\alpha_{0} ; Z\right)\right|=o_{p}\left(\alpha-\alpha_{0}\right)$ by a Taylor series expansion, and $\left|\hat{\Gamma}\left(\alpha_{0} ; Z\right)-\Gamma\left(\alpha_{0}\right)\right|=o_{p}(1)$ by law of large number. In light of the consistency of $\tilde{\alpha}$ and hence $\alpha^{*}, \hat{\Gamma}\left(\alpha^{*}, Z\right)$ converges to $\Gamma\left(\alpha_{0}\right)$ in probability. Therefore, $\sqrt{n}\left(\tilde{\alpha}-\alpha_{0}\right)$ is asymptotically normal with variance

$$
\tilde{V}_{\alpha}=\Gamma^{-1}\left(\alpha_{0}\right) C \Gamma^{-1}\left(\alpha_{0}\right)
$$

which is equal to

$$
-\Gamma^{-1}\left(\alpha_{0}\right)+\Gamma^{-1}\left(\alpha_{0}\right) \operatorname{var}\left\{q_{1}\left(\alpha_{0} ; Y_{i}\right)\right\} \Gamma^{-1}\left(\alpha_{0}\right)
$$


by noting $\operatorname{var}\left[I\left\{W_{i} \in(a, b)\right\} \phi\left(\alpha_{0} ; Z_{i}\right)\right]=-\Gamma\left(\alpha_{0}\right)$. Since

$$
\begin{aligned}
\operatorname{var}\left\{\phi\left(\alpha_{0} ; Z_{i}\right)\right\} & =E\left\{\phi^{\otimes 2}\left(\alpha_{0} ; Z_{i}\right)\right\} \geq_{p d} E\left[I\left\{W_{i} \in(a, b)\right\} \phi^{\otimes 2}\left(\alpha_{0} ; Z_{i}\right)\right] \\
& =\operatorname{var}\left[I\left\{W_{i} \in(a, b)\right\} \phi\left(\alpha_{0} ; Z_{i}\right)\right],
\end{aligned}
$$

where $\delta^{\otimes 2}=\delta \delta^{T}$, we have $-\Gamma^{-1}\left(\alpha_{0}\right)=\left(\operatorname{var}\left[I\left\{W_{i} \in(a, b)\right\} \phi\left(\alpha_{0} ; Z_{i}\right)\right]\right)^{-1} \geq_{p d}\left[\operatorname{var}\left\{\phi\left(\alpha_{0} ; Z_{i}\right)\right\}\right]^{-1}=$ $V_{\alpha}$. Thus $\tilde{V}_{\alpha}>_{p d} V_{\alpha}$.

The large sample properties of $\widetilde{\theta}_{p}$ then follow by an application of the functional delta method.

Theorem 3. $\sqrt{n}\left\{\widetilde{\theta}_{p}(v)-\theta(v)\right\}$ converges to a Gaussian process $\widetilde{\mathbb{Z}}_{p}$ with covariance structure $\Lambda\left(v_{1}, v_{2} ; \widetilde{V}_{\alpha}\right)$.

And the following theorem follows from $\tilde{V}_{\alpha}>_{p d} V_{\alpha}$.

Theorem 4. $\Lambda\left(v_{1}, v_{2} ; \widetilde{V}_{\alpha}\right)>_{p d} \Lambda\left(v_{1}, v_{2} ; V_{\alpha}\right.$. 


\section{Table 1}

Relative efficiencies of $\hat{\theta}_{p}, \tilde{\theta}_{p}$, and $\hat{\theta}_{n p}$ when the logistic model holds: $\operatorname{logit} 0[D=1 \mid F(Y)=v, T]=\alpha_{1}+\alpha_{2} R(v)+\alpha_{3} T+\alpha_{4} R(v) T$.

\begin{tabular}{|c|c|c|c|c|c|c|c|c|c|c|c|c|}
\hline \multirow[t]{2}{*}{ Setting } & \multirow[t]{2}{*}{$\alpha$} & \multirow[t]{2}{*}{$R(v)$} & & \multicolumn{9}{|c|}{$v$} \\
\hline & & & & 0.1 & 0.2 & 0.3 & 0.4 & 0.5 & 0.6 & 0.7 & 0.8 & 0.9 \\
\hline \multirow[t]{2}{*}{1} & $(0,1,1,1)$ & $v$ & $\operatorname{RE}\left(\hat{\theta}_{p}, \hat{\theta}_{n p}\right)$ & 1.33 & 1.36 & 1.28 & 1.20 & 1.17 & 1.17 & 1.17 & 1.16 & 1.11 \\
\hline & & & $\operatorname{RE}\left(\tilde{\theta}_{p}, \hat{\theta}_{n p}\right)$ & 1.31 & 1.34 & 1.26 & 1.19 & 1.16 & 1.15 & 1.16 & 1.14 & 1.10 \\
\hline \multirow[t]{2}{*}{2} & $(0,1,1,1)$ & $\Phi^{-1}(v)$ & $\operatorname{RE}\left(\hat{\theta}_{p}, \hat{\theta}_{n p}\right)$ & 1.64 & 1.67 & 1.62 & 1.55 & 1.48 & 1.42 & 1.36 & 1.30 & 1.24 \\
\hline & & & $\operatorname{RE}\left(\hat{\theta}_{p}, \hat{\theta}_{n p}\right)$ & 1.24 & 1.28 & 1.29 & 1.28 & 1.27 & 1.25 & 1.22 & 1.17 & 1.13 \\
\hline & $(0,1,1,1)$ & $10 \Phi^{-1}(v)$ & $\operatorname{RE}\left(\hat{\theta}_{p}, \hat{\theta}_{n p}\right)$ & 3.84 & 3.58 & 3.35 & 2.89 & 2.28 & 2.04 & 2.03 & 2.08 & 2.16 \\
\hline & & & $\operatorname{RE}\left(\tilde{\theta}_{p}, \hat{\theta}_{n p}\right)$ & 1.49 & 1.35 & 1.23 & 1.19 & 1.22 & 1.24 & 1.25 & 1.28 & 1.32 \\
\hline \multirow[t]{2}{*}{4} & $(0,1,1,1)$ & $-\log (v)$ & $\operatorname{RE}\left(\hat{\theta}_{p}, \hat{\theta}_{n p}\right)$ & 1.58 & 1.69 & 1.52 & 1.35 & 1.23 & 1.16 & 1.12 & 1.11 & 1.10 \\
\hline & & & $\operatorname{RE}\left(\tilde{\theta}_{p}, \hat{\theta}_{n p}\right)$ & 1.49 & 1.59 & 1.45 & 1.29 & 1.18 & 1.12 & 1.08 & 1.06 & 1.05 \\
\hline \multirow[t]{2}{*}{5} & $(0,0,10,8)$ & $\Phi^{-1}(v)$ & $\operatorname{RE}\left(\hat{\theta}_{p}, \hat{\theta}_{n p}\right)$ & 2.56 & 1.71 & 1.42 & 1.29 & 1.22 & 1.18 & 1.15 & 1.11 & 1.07 \\
\hline & & & $\operatorname{RE}\left(\tilde{\theta}_{p}, \hat{\theta}_{n p}\right)$ & 1.57 & 1.70 & 1.42 & 1.29 & 1.22 & 1.18 & 1.15 & 1.11 & 1.07 \\
\hline \multirow[t]{2}{*}{6} & $(0,3,20,15)$ & $\Phi^{-1}(v)$ & $\operatorname{RE}\left(\hat{\theta}_{p}, \hat{\theta}_{n p}\right)$ & 6.47 & 4.23 & 2.34 & 1.86 & 1.79 & 1.87 & 1.95 & 2.00 & 2.08 \\
\hline & & & $\operatorname{RE}\left(\tilde{\theta}_{p}, \hat{\theta}_{n p}\right)$ & 1.25 & 3.42 & 1.89 & 1.41 & 1.27 & 1.25 & 1.25 & 1.27 & 1.31 \\
\hline \multirow[t]{2}{*}{7} & $(1,2,1,0)$ & $\Phi^{-1}(v)$ & $\operatorname{RE}\left(\hat{\theta}_{p}, \hat{\theta}_{n p}\right)$ & 1.59 & 1.68 & 1.69 & 1.68 & 1.68 & 1.66 & 1.63 & 1.61 & 1.60 \\
\hline & & & $\operatorname{RE}\left(\tilde{\theta}_{p}, \hat{\theta}_{n p}\right)$ & 1.24 & 1.29 & 1.28 & 1.27 & 1.26 & 1.25 & 1.23 & 1.22 & 1.22 \\
\hline
\end{tabular}


Table 2

Results of simulation studies. ASD, standard deviation calculated using asymptotic theory; SD, empirical standard deviation across simulated data sets ; SE, average of estimated standard errors; CP, coverage probability of the 95\% Wald confidence interval.

\begin{tabular}{|c|c|c|c|c|c|c|c|c|c|c|c|c|c|c|c|c|c|}
\hline \multirow[t]{2}{*}{$R(v)$} & \multirow[t]{2}{*}{$v$} & \multirow[t]{2}{*}{$\theta$} & \multicolumn{5}{|c|}{$\hat{\theta}_{n p}$} & \multicolumn{5}{|c|}{$\hat{\theta}_{p}$} & \multicolumn{5}{|c|}{$\tilde{\theta}_{p}$} \\
\hline & & & est & ASD & SD & $\mathrm{SE}$ & $\mathrm{CP}$ & est & ASD & SD & $\mathrm{SE}$ & $\mathrm{CP}$ & est & ASD & SD & SE & $\mathrm{CP}$ \\
\hline \multirow[t]{9}{*}{$v$} & 0.1 & 0.844 & 0.844 & 0.035 & 0.034 & 0.036 & 0.966 & 0.845 & 0.030 & 0.031 & 0.030 & 0.951 & 0.845 & 0.030 & 0.031 & 0.030 & 0.947 \\
\hline & 0.2 & 0.819 & 0.819 & 0.036 & 0.036 & 0.036 & 0.957 & 0.820 & 0.031 & 0.031 & 0.030 & 0.943 & 0.820 & 0.031 & 0.032 & 0.031 & 0.942 \\
\hline & 0.3 & 0.794 & 0.794 & 0.037 & 0.038 & 0.038 & 0.953 & 0.794 & 0.033 & 0.034 & 0.033 & 0.942 & 0.794 & 0.033 & 0.034 & 0.033 & 0.947 \\
\hline & 0.4 & 0.768 & 0.768 & 0.038 & 0.041 & 0.039 & 0.946 & 0.767 & 0.035 & 0.037 & 0.035 & 0.939 & 0.767 & 0.035 & 0.037 & 0.035 & 0.940 \\
\hline & 0.5 & 0.742 & 0.740 & 0.040 & 0.042 & 0.041 & 0.944 & 0.741 & 0.037 & 0.039 & 0.037 & 0.935 & 0.741 & 0.037 & 0.039 & 0.037 & 0.936 \\
\hline & 0.6 & 0.716 & 0.715 & 0.042 & 0.043 & 0.042 & 0.941 & 0.714 & 0.039 & 0.041 & 0.039 & 0.932 & 0.714 & 0.039 & 0.041 & 0.039 & 0.936 \\
\hline & 0.7 & 0.691 & 0.690 & 0.043 & 0.045 & 0.044 & 0.942 & 0.689 & 0.040 & 0.042 & 0.041 & 0.933 & 0.689 & 0.040 & 0.042 & 0.041 & 0.932 \\
\hline & 0.8 & 0.666 & 0.665 & 0.045 & 0.047 & 0.046 & 0.940 & 0.664 & 0.042 & 0.044 & 0.042 & 0.937 & 0.664 & 0.042 & 0.044 & 0.042 & 0.937 \\
\hline & 0.9 & 0.643 & 0.640 & 0.047 & 0.048 & 0.047 & 0.946 & 0.640 & 0.045 & 0.046 & 0.045 & 0.938 & 0.640 & 0.045 & 0.046 & 0.045 & 0.938 \\
\hline \multirow[t]{9}{*}{$\Phi(v)$} & 0.1 & 0.654 & 0.651 & 0.046 & 0.050 & 0.047 & 0.935 & 0.653 & 0.036 & 0.038 & 0.036 & 0.931 & 0.652 & 0.042 & 0.044 & 0.041 & 0.926 \\
\hline & 0.2 & 0.655 & 0.652 & 0.045 & 0.047 & 0.046 & 0.935 & 0.654 & 0.034 & 0.037 & 0.035 & 0.933 & 0.652 & 0.039 & 0.042 & 0.039 & 0.930 \\
\hline & 0.3 & 0.647 & 0.647 & 0.043 & 0.045 & 0.044 & 0.948 & 0.647 & 0.034 & 0.035 & 0.033 & 0.939 & 0.645 & 0.038 & 0.040 & 0.038 & 0.938 \\
\hline & 0.4 & 0.632 & 0.630 & 0.042 & 0.045 & 0.043 & 0.946 & 0.631 & 0.033 & 0.035 & 0.033 & 0.940 & 0.630 & 0.037 & 0.039 & 0.037 & 0.938 \\
\hline & 0.5 & 0.611 & 0.610 & 0.041 & 0.044 & 0.043 & 0.947 & 0.610 & 0.034 & 0.035 & 0.034 & 0.951 & 0.609 & 0.037 & 0.039 & 0.037 & 0.932 \\
\hline & 0.6 & 0.586 & 0.587 & 0.042 & 0.044 & 0.043 & 0.947 & 0.585 & 0.035 & 0.037 & 0.035 & 0.937 & 0.584 & 0.038 & 0.040 & 0.038 & 0.932 \\
\hline & 0.7 & 0.560 & 0.560 & 0.044 & 0.046 & 0.044 & 0.936 & 0.559 & 0.037 & 0.039 & 0.038 & 0.941 & 0.559 & 0.040 & 0.042 & 0.040 & 0.934 \\
\hline & 0.8 & 0.536 & 0.535 & 0.046 & 0.048 & 0.046 & 0.942 & 0.535 & 0.040 & 0.042 & 0.040 & 0.940 & 0.534 & 0.042 & 0.045 & 0.042 & 0.937 \\
\hline & 0.9 & 0.514 & 0.513 & 0.048 & 0.051 & 0.048 & 0.934 & 0.513 & 0.043 & 0.044 & 0.043 & 0.944 & 0.513 & 0.045 & 0.048 & 0.045 & 0.941 \\
\hline
\end{tabular}




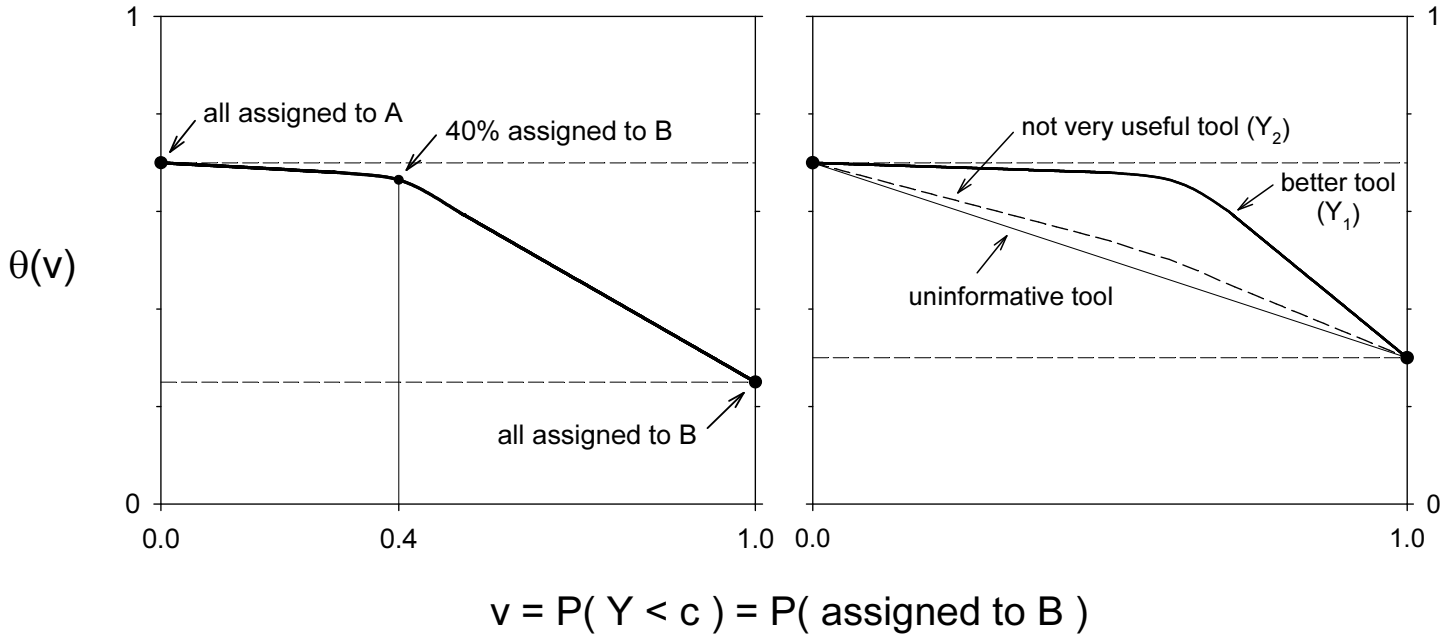

Figure 1. A schematic diagram of the selection impact (SI) curve, $\theta(v)=P\{D=1 \mid(y>$ $c, T=1)$ or $(Y<c, T>0)\}$. 


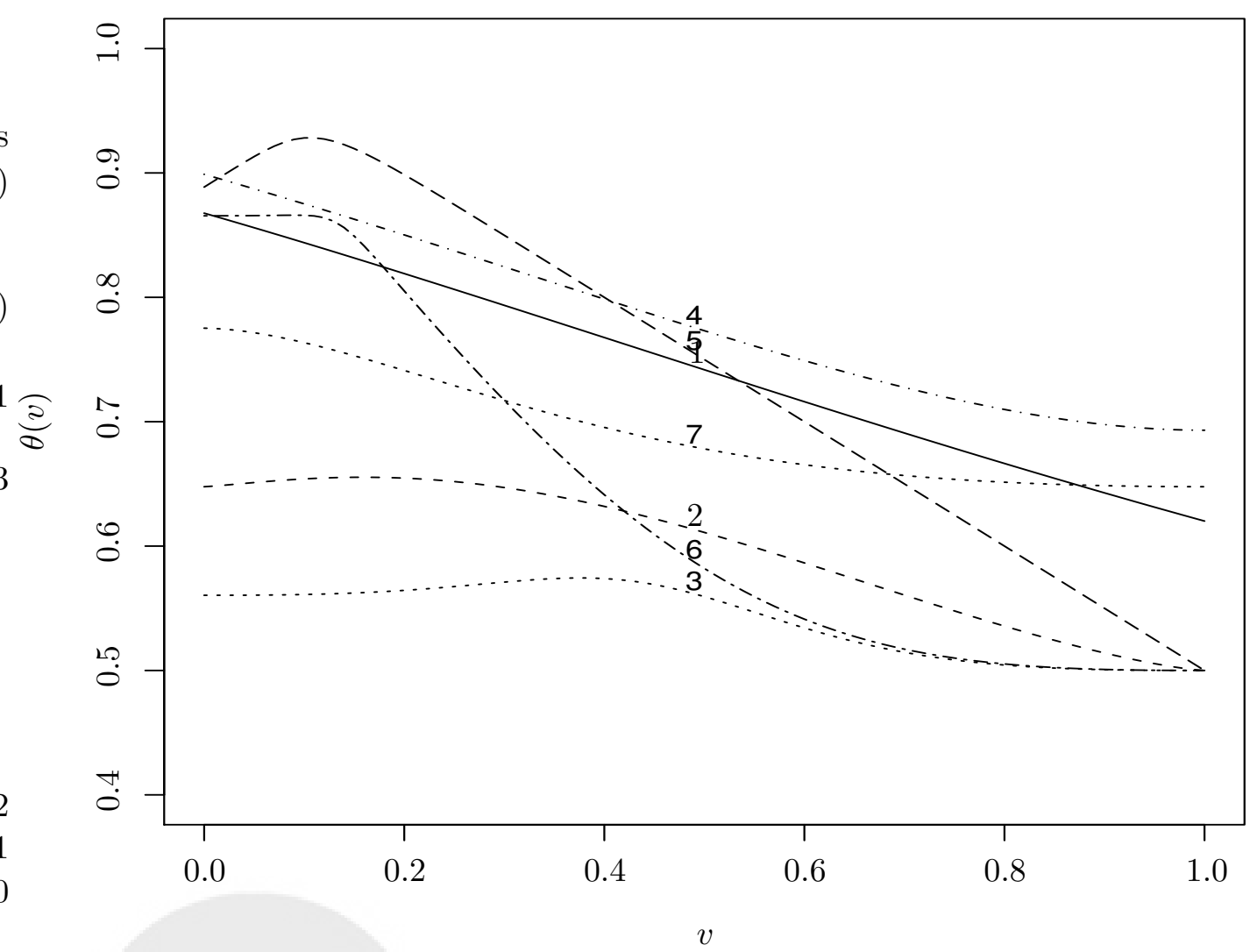

Figure 2. $\theta(v)$ for the settings described in Table 1. 


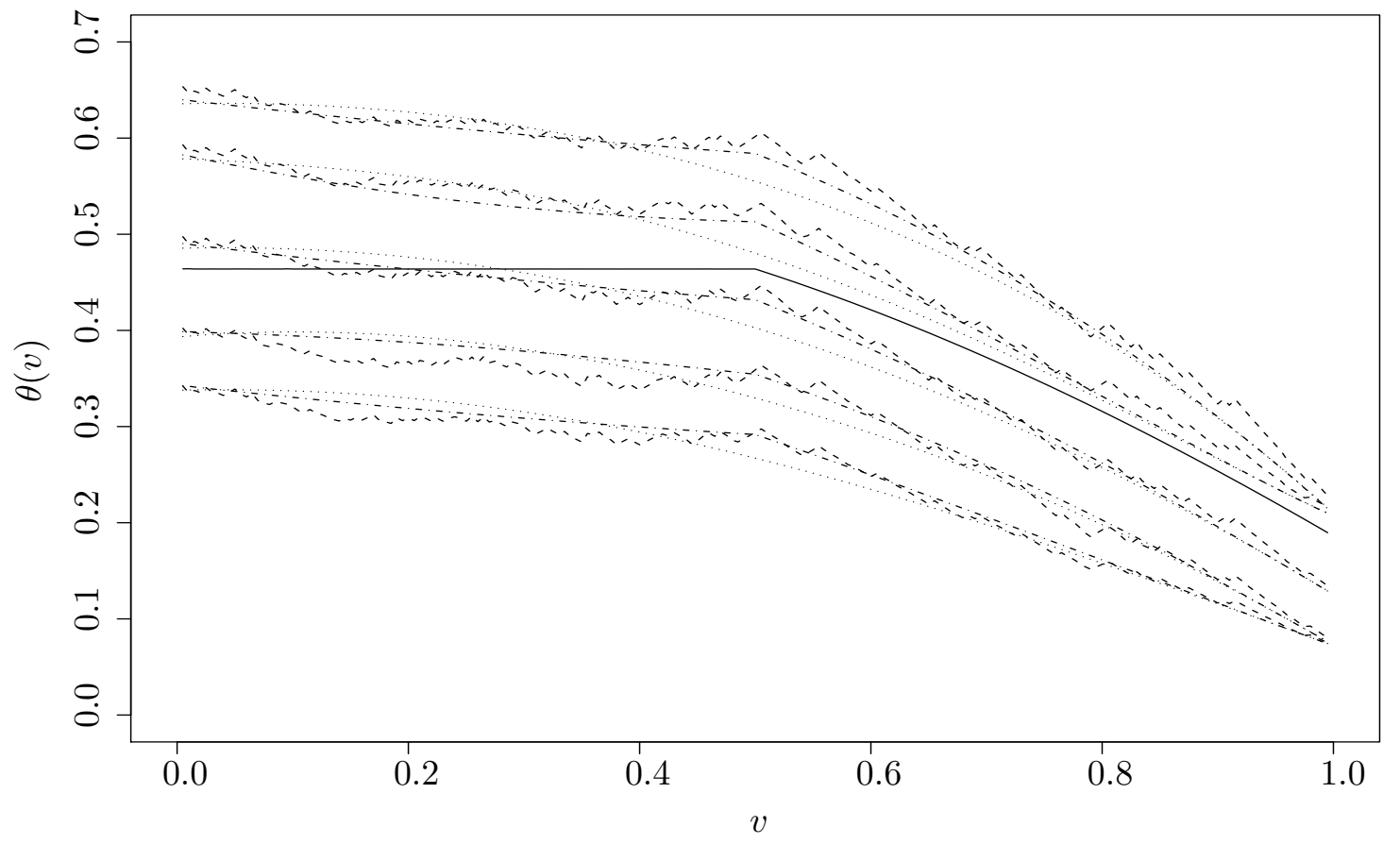

Figure 3. The SI curve estimated from the Carpal Tunnel Syndrome study. True $\theta(v)$, solid curve; nonparametric estimate $\hat{\theta}_{n p}$, dashed curve; parametric estimate $\tilde{\theta}_{p}$, dash-dotted curve; misspecified parametric estimate $\tilde{\theta}_{p}^{*}$, dotted curve. $95 \%$ confidence bands are shown with the outer curves, $95 \%$ pointwise confidence intervals are shown with the intermediate curves, the estimates themselves are shown with the center curves. 


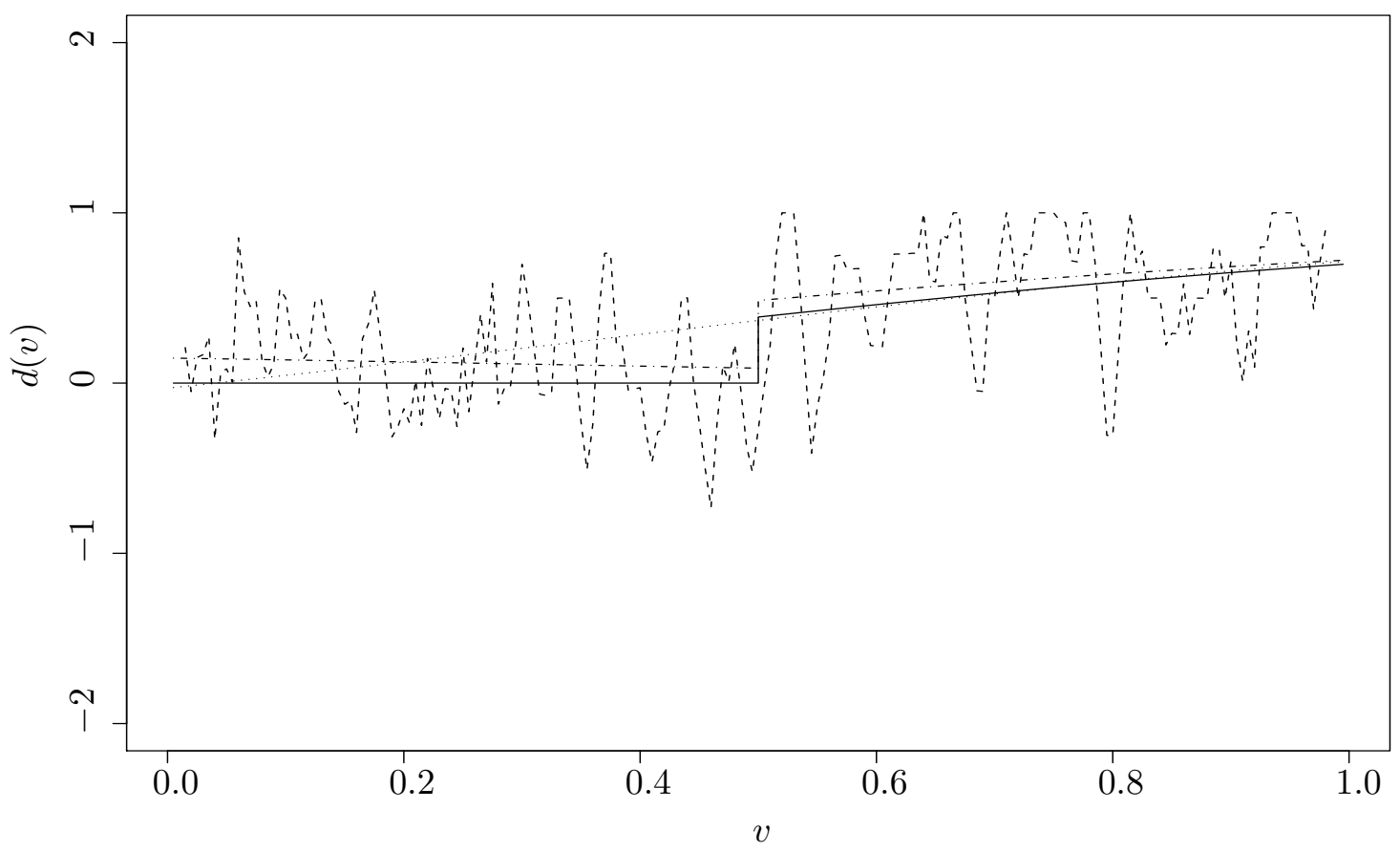

Figure 4. $d(v)=P\left[D=1 \mid Y=y_{v}, T=1\right]-P\left[D=1 \mid Y=y_{v}, T=0\right]$ estimated from the Carpal Tunnel Syndrome study. Truth, solid curve; nonparametric estimate, dashed curve; parametric estimate, dash-dotted curve; misspecified parametric estimate, dotted curve. 